\title{
The 2020 release of the ExoMol database: Molecular line lists for exoplanet and other hot atmospheres
}

\author{
Jonathan Tennyson $^{\mathrm{a}, *}$, Sergei N. Yurchenko ${ }^{a}$, Ahmed F. Al-Refaie ${ }^{a}$, Victoria H.J. Clark ${ }^{\mathrm{a}}$, \\ Katy L. Chubb ${ }^{\mathrm{a}, \mathrm{b}}$, Eamon K. Conway ${ }^{\mathrm{a}, \mathrm{i}}$, Akhil Dewan ${ }^{\mathrm{a}}$, Maire N. Gorman ${ }^{\mathrm{a}, \mathrm{c}}$, Christian Hilla,d, \\ A.E. Lynas-Gray a,g,h, Thomas Mellor ${ }^{\mathrm{a}}$, Laura K. McKemmish ${ }^{\mathrm{a}, \mathrm{e}}$, Alec Owens ${ }^{\mathrm{a}}$, \\ Oleg L. Polyansky ${ }^{a, j}$, Mikhail Semenov ${ }^{a}$, Wilfrid Somogyi ${ }^{a}$, Giovanna Tinetti ${ }^{a}$, \\ Apoorva Upadhyay ${ }^{a}$, Ingo Waldmann ${ }^{a}$, Yixin Wang ${ }^{\text {a,f }}$, Samuel Wright ${ }^{a}$, Olga P. Yurchenko ${ }^{a}$ \\ a Department of Physics and Astronomy, University College London, London, WC1E 6BT, UK \\ b SRON Netherlands Institute for Space Research, Sorbonnelaan 2, 3584 CA, Utrecht, Netherlands \\ ${ }^{c}$ Department of Physics, Aberystwyth University, Ceredigion, UK, SY23 3BZ, UK \\ ${ }^{\mathrm{d}}$ Nuclear Data Section, International Atomic Energy Agency, Vienna A-1400, Austria \\ e School of Chemistry, University of New South Wales, Sydney 2052, Australia \\ ${ }^{\mathrm{f}}$ Nankai University, 94 Weijin Road, Tianjin, China \\ ${ }^{g}$ Department of Physics, University of Oxford, England, United Kingdom \\ ${ }^{\mathrm{h}}$ Department of Physics and Astronomy, University of the Western Cape, South Africa \\ ${ }^{\mathrm{i}}$ Atomic and Molecular Physics Division, Center for Astrophysics | Harvard \& Smithsonian, Cambridge, MA USA \\ ${ }^{\mathrm{j}}$ Institute of Applied Physics, Russian Academy of Sciences, Ul'yanov Street 46, Nizhny Novgorod, 603950, Russia
}

\section{A R T I C L E I N F O}

Article history:

Received 10 June 2020

Revised 21 July 2020

Accepted 22 July 2020

Available online 24 July 2020

\section{Keywords:}

Infrared

Visible

Einstein A coefficients

Transition frequencies

Partition functions

Cooling functions

Lifetimes

Cross sections

$k$ Coefficients

Landé $g$-factors

pressure broadening

\begin{abstract}
A B S T R A C T
The ExoMol database (www.exomol.com) provides molecular data for spectroscopic studies of hot atmospheres. While the data are intended for studies of exoplanets and other astronomical bodies, the dataset is widely applicable. The basic form of the database is extensive line lists; these are supplemented with partition functions, state lifetimes, cooling functions, Landé g-factors, temperature-dependent cross sections, opacities, pressure broadening parameters, $k$-coefficients and dipoles. This paper presents the latest release of the database which has been expanded to consider 80 molecules and 190 isotopologues totaling over 700 billion transitions. While the spectroscopic data are concentrated at infrared and visible wavelengths, ultraviolet transitions are being increasingly considered in response to requests from observers. The core of the database comes from the ExoMol project which primarily uses theoretical methods, albeit usually fine-tuned to reproduce laboratory spectra, to generate very extensive line lists for studies of hot bodies. The data have recently been supplemented by line lists derived from direct laboratory observations, albeit usually with the use of ab initio transition intensities. A major push in the new release is towards accurate characterisation of transition frequencies for use in high resolution studies of exoplanets and other bodies.
\end{abstract}

(c) 2020 Elsevier Ltd. All rights reserved.

\section{Introduction}

The ExoMol project was started in 2011 [1] with the purpose of providing molecular line lists for studies of exoplanets and other (hot) atmospheres. Besides data demands for exoplanets [2], other hot astronomical bodies with significant molecular content in their atmospheres include cool stars [3] and brown dwarfs [3,4]. On Earth, similar spectroscopic data are required to study flames $[5,6]$,

\footnotetext{
* Corresponding author.

E-mail address: j.tennyson@ucl.ac.uk (J. Tennyson).
}

discharge plasmas [7], explosions [8] and the gases emitted from smoke stacks [9]. In addition, bodies in non-local thermodynamic equilibrium (non-LTE) such as comets and masers give observable emissions from highly excited states [10-12].

ExoMol data are proving very popular among the exoplanet atmospheric modellers owing to their extensive coverage of the molecular species and their completeness as a function of both frequency and, very importantly, temperature. ExoMol data have been incorporated into many radiative transfer and retrieval codes including Tau-REx [13-15], Phoenix [16], NEMESIS [17], CHIMERA [18], HELIOS [19] and HELIOS-r2 [20], ATMO [21-23], ARCiS [24], 
ARTES [25], HyDRA [26], GENESIS [27], petitRADTRANS [28], PLATON [29], VSTAR [30,31], BART [32] and Pyrat Bay [33]. In addition the ExoMol database has been used to support a variety of other studies. Examples including the analysis of gaseous ammonia in Jupiter [34], tentative detection of $\mathrm{H}_{2} \mathrm{~S}$ in Uranus [35], detection of $\mathrm{CaO}$ in meteors [36] and $\mathrm{SiO}$ in $\mathrm{B}[\mathrm{e}]$ Supergiants [37], Late-type stars [38] or circumstellar environment [39]. Detections in the atmospheres of exoplanets are discussed below. More diverse applications include modelling laser analysis of Solar System objects $[40,41]$, isotope abundance quantification in stars [42], search for molecules highly sensitive to the proton-to-electron mass ratio variation [43,44], models of molecular steering [45,46], design of $\mathrm{THz}$ lasers [47], design of dark matter detection schemes [48] and the design of novel propulsion systems [49].

A number of diatomic molecules for which ExoMol provides extensive hot line lists have been detected in sunspots. These include $\mathrm{SiO}$ [50], $\mathrm{SiH}$ [51], SH [52], $\mathrm{BeH}$ [53], VO [54], as well as $\mathrm{MgH}, \mathrm{MgO}$, $\mathrm{TiO}, \mathrm{C}_{2}$ and $\mathrm{CaH}$ which have all long been observed in sunspots [55]. In addition the spectrum of water is well-known in sunspots [56-60]; studies of water spectra in sunspots should be aided by the new POKAZATEL water line list [61] which is designed for studies at temperatures above $3000 \mathrm{~K}$ such as those encountered in solar umbra and penumbra.

ExoMol line lists have been published as a series in the journal Monthly Notices of the Royal Astronomical Society (see below) and a first data release in 2016 [62], henceforth ExoMol2016, provided full documentation for the ExoMol database which can be found at www.exomol.com. The basic form of the database is extensive line lists; these are supplemented with partition functions, state lifetimes, cooling functions, Landé g-factors, temperaturedependent cross sections, opacities, pressure broadening parameters, $k$-coefficients and dipoles. The ExoMol website also offers an extensive bibliography database on research literature on molecules relevant for ExoMol applications. So far the bibliography database contains 6709 sources.

Since ExoMol started there have been major changes in exoplanet science which have driven further expansion and development of the ExoMol database. The first is the discovery of rocky exoplanets orbiting so near to their host star that their surfaces are likely to be molten. The atmospheres of these planets are thought to contain a variety of species not considered previously [63-66]. Secondly higher temperature planets have been observed than anyone anticipated, the poster child for hot exoplanets is Kelt$9 \mathrm{~b}$ which is thought to reach temperatures of $4000 \mathrm{~K}$ [67], hotter than most of the stars in our Galaxy! This has led to the need to construct line list for key species over an extended temperature range. Third is the development of Doppler-shift high resolution spectroscopy of exoplanets $[68,69]$ which has proved a powerful tool for detecting molecules but fails in the absence of highly accurate molecular line lists [70]. These developments have led us to both expand the range of molecules included in the database and to begin a systematic attempt to improve the accuracy of the line positions for the line lists contained in the database. Progress on both of these objectives is described below as well as work on extending the coverage of the database into the ultraviolet.

Of course ExoMol is not the only database providing spectroscopic data for atmospheric studies. For the Earth's atmosphere, databases HITRAN [71-73] and GEISA [74,75] provide comprehensive and validated datasets for approximately 50 key molecules. However, these databases are designed for studies at room temperature and below and do not contain the necessary data for adequately calculating radiative transport in hot bodies. The HITEMP database was constructed to extend HITRAN to higher temperatures. The latest, 2010, release [76] only contained data on 5 molecules $\left(\mathrm{OH}, \mathrm{NO}, \mathrm{CO}, \mathrm{H}_{2} \mathrm{O}\right.$ and $\left.\mathrm{CO}_{2}\right)$ and improved hot line lists are available for all these molecules; an update to HITEMP is currently in progress [77-79]. Other relevant databases include TheoReTS [80], which contains hot line lists for 8 polyatomic molecules, and Kurucz's compilation of data, which is very complete for atomic sources but contains data only on about ten diatomic molecules [81] all of which are covered by ExoMol, usually to higher accuracy. The MoLLIST data base of Bernath and coworkers [82] contains empirically derived line lists for about 20 diatomic species. These line lists have recently been incorporated into the ExoMol database [83], see Table 2 below. Databases of hot molecular spectra for other specialised applications include those for combustion [6,84] and studies of laser-induced plasmas [85]. ExoMol line lists together with data from other sources will serve as the main data source for exoplanet studies planned for the upcoming space missions Ariel and JWST.

The methodology [65,86-91] and software [92,93] developed and used by the ExoMol project has been extensively documented elsewhere. Here we will only consider those aspects which impinge on the new ExoMol2020 release.

\section{Database coverage}

The ExoMol project aims at complete coverage of the spectroscopic properties of molecules which are deemed to be important in hot astrophysical environments. Coverage concerns (a) the molecular species considered, including isotopologues; (b) the spectroscopic and thermodynamic properties considered; (c) the frequency range considered and (d) the upper temperature range for which the data are reasonably complete. Both the required temperature and frequency range completeness are to some extent a judgement on what is required for astronomical and other studies.

Unlike ExoMol2016, the ExoMol2020 database essentially provides a complete dataset for modelling hot atmospheres. While new species are still being added, commonly at the request of users, the database now contains molecular data for 80 molecules and covers the key molecules thought to be important for exoplanetary studies. In many cases there is more than one line list for a given isotopologue. For this reason it is our practice to name each line list, including (unnamed) ones we have imported from other sources. In the case of multiple line lists the ExoMol website provides a recommended line list for the given species. This recommendation is for studies of hot atmospheres; other available line lists may be more suitable for room temperature studies.

For ease of understanding we have divided the description of line lists provided into three tables. Tables 1 and 2 summarise the molecules for which data are provided by ExoMol and give the characteristics of the line list in each case. Table 1 details line lists which have been formally published as part of the ExoMol project while Table 2 shows line lists imported from other sources which have been recast in the ExoMol format (see Wang et al [83] for example); these line lists are fully integrated into the database. Table 3 lists the main extra line lists available through the ExoMol website; this table is not comprehensive but the other line lists available on the ExoMol website and not listed in Table 1-3 are largely of historic interest only.

\section{Individual line lists}

An overview of the line lists in the ExoMol database is given in Fig. 1.

One general issue is that Medvedev and co-workers $[165,186]$ identified a numerical problem with the intensities of high overtone transitions computed with the standard compilation of the diatomic vibration-rotation program LEvel [187]. Our diatomic line lists computed with LEVEL or Duo [188] have been 
Table 1

Datasets created by the ExoMol project and included in the ExoMol database.

\begin{tabular}{|c|c|c|c|c|c|c|c|c|}
\hline Paper & Molecule & $N_{\text {iso }}$ & $T_{\max }$ & $N_{\text {elec }}$ & $N_{\text {lines }}{ }^{a}$ & DSName & & Reference \\
\hline I & $\mathrm{BeH}$ & 1 & 2000 & 1 & 16400 & Yadin & & Yadin et al. [94] \\
\hline I & $\mathrm{MgH}$ & 3 & 2000 & 1 & 10354 & Yadin & $\boldsymbol{v}$ & Yadin et al. [94] \\
\hline I & $\mathrm{CaH}$ & 1 & 2000 & 1 & 15278 & Yadin & $\boldsymbol{v}$ & Yadin et al. [94] \\
\hline II & $\mathrm{SiO}$ & 5 & 9000 & 1 & 254675 & EJBT & $\boldsymbol{v}$ & Barton et al. [95] \\
\hline III & $\mathrm{HCN} / \mathrm{HNC}$ & $1^{a}$ & 4000 & 1 & 399000000 & Harris & $\boldsymbol{v}$ & Barber et al. [96] \\
\hline IV & $\mathrm{CH}_{4}$ & 1 & 2000 & 1 & 34153806005 & YT34to10 & $\boldsymbol{v}$ & Yurchenko and Tennyson [97], Yurchenko et al. [98] \\
\hline $\mathrm{V}$ & $\mathrm{NaCl}$ & 2 & 3000 & 1 & 702271 & Barton & $\boldsymbol{v}$ & Barton et al. [99] \\
\hline V & $\mathrm{KCl}$ & 4 & 3000 & 1 & 1326765 & Barton & $\boldsymbol{v}$ & Barton et al. [99] \\
\hline VI & PN & 2 & 5000 & 1 & 142512 & YYLT & $\boldsymbol{v}$ & Yorke et al. [100] \\
\hline VII & $\mathrm{PH}_{3}$ & 1 & 1500 & 1 & 16803703395 & SAITY & $\boldsymbol{r}$ & Sousa-Silva et al. [101] \\
\hline VIII & $\mathrm{H}_{2} \mathrm{CO}$ & 1 & 1500 & 1 & 10000000000 & AYTY & $\boldsymbol{v}$ & Al-Refaie et al. [102] \\
\hline IX & AlO & 4 & 8000 & 3 & 4945580 & ATP & $\boldsymbol{v}$ & Patrascu et al. [103] \\
\hline $\mathrm{X}$ & $\mathrm{NaH}$ & 2 & 7000 & 2 & 79898 & Rivlin & $\checkmark$ & Rivlin et al. [104] \\
\hline XI & $\mathrm{HNO}_{3}$ & 1 & 500 & 1 & 6722136109 & AljS & $\boldsymbol{v}$ & Pavlyuchko et al. [105] \\
\hline XII & $\mathrm{CS}$ & 8 & 3000 & 1 & 548312 & JnK & $\boldsymbol{v}$ & Paulose et al. [106] \\
\hline XIII & $\mathrm{CaO}$ & 1 & 5000 & 5 & 21279299 & VBATHY & $\boldsymbol{v}$ & Yurchenko et al. [107] \\
\hline XIV & $\mathrm{SO}_{2}$ & 1 & 2000 & 1 & 1300000000 & ExoAmes & $\boldsymbol{v}$ & Underwood et al. [108] \\
\hline$X V$ & $\mathrm{H}_{2} \mathrm{O}_{2}$ & 1 & 1250 & 1 & 20000000000 & APTY & $\boldsymbol{v}$ & Al-Refaie et al. [109] \\
\hline XVI & $\mathrm{H}_{2} \mathrm{~S}$ & 1 & 2000 & 1 & 1155303730 & AYT2 & $\boldsymbol{v}$ & Azzam et al. [110] \\
\hline XVII & $\mathrm{SO}_{3}$ & 1 & 800 & 1 & 21000000000 & UYT2 & $\boldsymbol{v}$ & Underwood et al. [111] \\
\hline XVIII & Vo & 1 & 2000 & 13 & 277131624 & VOMYT & $\boldsymbol{r}$ & McKemmish et al. [112] \\
\hline XIX & $\mathrm{H}_{2}{ }^{17,18} \mathrm{O}$ & 2 & 3000 & 1 & 519461789 & HotWat78 & $\boldsymbol{v}$ & Polyansky et al. [113] \\
\hline $\mathrm{XX}$ & $\mathrm{H}_{3}^{+}$ & $1^{b}$ & 3000 & 1 & 11500000000 & MiZATeP & $\boldsymbol{v}$ & Mizus et al. [114] \\
\hline XXI & NO & 6 & 5000 & 2 & 2281042 & NOName & $\boldsymbol{v}$ & Wong et al. [115] \\
\hline XXII & $\mathrm{SiH}_{4}$ & 1 & 1200 & 1 & 62690449078 & OY2T & $\boldsymbol{v}$ & Owens et al. [116] \\
\hline XXIII & $\mathrm{PO}$ & 1 & 5000 & 1 & 2096289 & POPS & $\boldsymbol{v}$ & Prajapat et al. [117] \\
\hline XXIII & PS & 1 & 5000 & 3 & 30394544 & POPS & $\boldsymbol{v}$ & Prajapat et al. [117] \\
\hline XXIV & $\mathrm{SiH}$ & 4 & 5000 & 3 & 1724841 & SiGHTLY & $\boldsymbol{v}$ & Yurchenko et al. [118] \\
\hline $\mathrm{XXV}$ & SiS & 12 & 5000 & 1 & 91715 & UCTY & $\boldsymbol{v}$ & Upadhyay et al. [119] \\
\hline XXVI & NS & 6 & 5000 & 1 & 3479067 & $\mathrm{SNaSH}$ & $\boldsymbol{v}$ & Yurchenko et al. [120] \\
\hline XXVI & HS & 6 & 5000 & 1 & 219463 & $\mathrm{SNaSH}$ & & Yurchenko et al. [120] \\
\hline XXVII & $\mathrm{C}_{2} \mathrm{H}_{4}$ & 1 & 700 & 1 & 60000000000 & MaYTY & $\boldsymbol{v}$ & Mant et al. [121] \\
\hline XXVIII & $\mathrm{AlH}$ & 3 & 5000 & 3 & 40000 & AlHambra & $\boldsymbol{r}$ & Yurchenko et al. [122] \\
\hline XXIX & $\mathrm{CH}_{3} \mathrm{Cl}$ & 2 & 1200 & 1 & 166279593333 & OYT & $\boldsymbol{v}$ & Owens et al. [123] \\
\hline $\mathrm{XXX}$ & $\mathrm{H}_{2}{ }^{16} \mathrm{O}$ & $1^{c}$ & 5000 & 1 & 5745071340 & POKAZATEL & $\boldsymbol{v}$ & Polyansky et al. [61] \\
\hline XXXI & $\mathrm{C}_{2}$ & 3 & 5000 & 8 & 6080920 & 8states & $\boldsymbol{v}$ & Yurchenko et al. [124] \\
\hline XXXII & $\mathrm{MgO}$ & 5 & 5000 & 5 & 72833173 & LiTY & $\boldsymbol{v}$ & Li et al. [125] \\
\hline XXXIII & $\mathrm{TiO}$ & 5 & 5000 & 13 & 59000000 & Toto & $\boldsymbol{v}$ & McKemmish et al. [126] \\
\hline XXXIV & $\mathrm{PH}$ & 1 & 4000 & 2 & 65055 & LaTY & $\boldsymbol{v}$ & Langleben et al. [127] \\
\hline XXXV & $\mathrm{NH}_{3}$ & $1^{d}$ & 1500 & 1 & 16900000 & CoYuTe & $\boldsymbol{v}$ & Coles et al. [128] \\
\hline XXXVI & $\mathrm{SH}$ & 2 & 3000 & 2 & 572145 & GYT & $\boldsymbol{v}$ & Gorman et al. [129] \\
\hline XXXVII & $\mathrm{HCCH}$ & 1 & 2000 & 1 & 4347381911 & aCeTY & $\boldsymbol{r}$ & Chubb et al. [130] \\
\hline XXXVIII & $\mathrm{SiO}_{2}$ & 1 & 3000 & 1 & 32951275437 & OYT3 & $\boldsymbol{v}$ & Owens et al. [131] \\
\hline XXXIX & $\mathrm{CO}_{2}$ & 1 & 3000 & 1 & 7996570390 & UCL-4000 & $\boldsymbol{v}$ & Yurchenko et al. [132] \\
\hline $\mathrm{XL}$ & $\mathrm{H}_{3} \mathrm{O}^{+}$ & 1 & 1500 & 1 & 2089331073 & eXeL & $\boldsymbol{v}$ & Yurchenko et al. [133] \\
\hline
\end{tabular}

Paper Number in series published in Mon. Not. R. Astron. Soc.; $N_{\text {iso }}$ Number of isotopologues considered; $T_{\max }$ Maximum temperature for which the line list is complete; $N_{\text {elec }}$ Number of electronic states considered; $N_{\text {lines }}$ Number of lines: value is for the main isotope. $\boldsymbol{V}$ indicates line list recommended for studies of hot atmospheres. ${ }^{a}$ The Larner line list for $\mathrm{H}^{13} \mathrm{CN} / \mathrm{HN}^{13} \mathrm{C}$ due to Harris et al. [134] is recommended. ${ }^{b}$ The ST line list for $\mathrm{H}_{2} \mathrm{D}^{+}$due to Sochi and Tennyson [135] is recommended,. ${ }^{c}$ The VTT line list for HDO due to Voronin et al. [136] is recommended. ${ }^{d}$ There is a room temperature

${ }^{15} \mathrm{NH}_{3}$ line list due to Yurchenko [137].

\begin{tabular}{|c|c|c|c|c|c|c|c|c|c|c|c|}
\hline $\mathrm{AlH}$ & $\mathrm{AlCl}$ & $\mathrm{AlO}$ & $\mathrm{BeH}$ & $\mathrm{C}_{2}$ & $\mathrm{CaO}$ & $\mathrm{CaF}$ & $\mathrm{CaH}$ & $\mathrm{CH}$ & $\mathrm{CN}$ & $\mathrm{CO}$ & $\mathrm{CP}$ \\
\hline $\mathrm{CrH}$ & $\mathrm{CS}$ & $\mathrm{FeH}$ & $\mathrm{FeO}$ & $\mathrm{H}_{2}$ & $\mathrm{H}_{3}^{+}$ & $\mathrm{H}_{3} \mathrm{O}^{+}$ & $\mathrm{HCl}$ & $\mathrm{HF}$ & $\mathrm{LiCl}$ & $\mathrm{LiF}$ & $\mathrm{LiH}$ \\
\hline $\mathrm{LiH}^{+}$ & $\mathrm{KCl}$ & $\mathrm{KF}$ & $\mathrm{MgF}$ & $\mathrm{MgH}$ & $\mathrm{MgO}$ & $\mathrm{N}_{2}$ & $\mathrm{NaCl}$ & $\mathrm{NaF}$ & $\mathrm{NaH}$ & $\mathrm{NaF}$ & $\mathrm{NiH}$ \\
\hline $\mathrm{NH}$ & $\mathrm{NS}$ & $\mathrm{NO}$ & $\mathrm{OH}$ & $\mathrm{O}_{2}$ & $\mathrm{PH}$ & $\mathrm{PN}$ & $\mathrm{PO}$ & $\mathrm{PS}$ & $\mathrm{ScH}$ & $\mathrm{SiC}$ & $\mathrm{SiH}$ \\
\hline $\mathrm{SiS}$ & $\mathrm{SH}$ & $\mathrm{SiO}$ & $\mathrm{SiS}$ & $\mathrm{SO}$ & $\mathrm{TiO}$ & $\mathrm{TiH}$ & $\mathrm{TiF}$ & $\mathrm{VO}$ & $\mathrm{VN}$ & $\mathrm{YO}$ & $\mathrm{ZnS}$ \\
\hline $\mathrm{C}_{3}$ & $\mathrm{CaOH}$ & $\mathrm{CO}_{2}$ & $\mathrm{CH}_{2}$ & $\mathrm{H}_{3}^{+}$ & $\mathrm{H}_{2} \mathrm{D}^{+}$ & $\mathrm{HCN}$ & $\mathrm{HNC}$ & $\mathrm{H}_{2} \mathrm{O}$ & $\mathrm{HDO}$ & $\mathrm{H}_{2} \mathrm{~S}$ & $\mathrm{KOH}$ \\
\hline $\mathrm{NaOH}^{\mathrm{N}}$ & $\mathrm{N}_{2} \mathrm{O}$ & $\mathrm{OCS}$ & $\mathrm{PO}_{2}$ & $\mathrm{SiH}_{2}$ & $\mathrm{SiO}_{2}$ & $\mathrm{SO}_{2}$ & $\mathrm{AsH}_{3}$ & $\mathrm{CH}_{3}$ & $\mathrm{C}_{2} \mathrm{H}_{2}$ & $\mathrm{HOOH}$ & $\mathrm{H}_{2} \mathrm{CO}$ \\
\hline $\mathrm{NH}_{3}$ & $\mathrm{SiH}_{3}$ & $\mathrm{PH}_{3}$ & $\mathrm{P}_{2} \mathrm{H}_{2}$ & $\mathrm{PF}_{3}$ & $\mathrm{SO}_{3}$ & $\mathrm{CH}_{4}$ & $\mathrm{CH}_{3} \mathrm{D}$ & $\mathrm{CH}_{3} \mathrm{Cl}$ & $\mathrm{CH}_{3} \mathrm{~F}$ & $\mathrm{SiH}_{4}$ & $\mathrm{HNO}_{3}$ \\
\hline $\mathrm{C}_{2} \mathrm{H}_{4}$ & $\mathrm{C}_{2} \mathrm{H}_{6}$ & $\mathrm{C}_{3} \mathrm{H}_{8}$ & & & & & & & & & \\
\hline
\end{tabular}

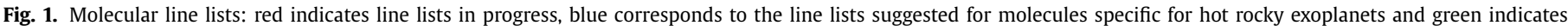
line lists which contain data applicable for high resolution. 
Table 2

Datasets not created as part of the ExoMol project but included in the ExoMol database.

\begin{tabular}{|c|c|c|c|c|c|c|c|c|}
\hline Molecule & $N_{\text {iso }}$ & $T_{\max }$ & $N_{\text {elec }}$ & $N_{\text {lines }}$ & DSName & & Reference & Methodology \\
\hline $\mathrm{H}_{2}$ & 1 & 10000 & 1 & 4712 & RACPPK & $\checkmark$ & Roueff et al. [138] & $\mathrm{Ab}$ initio \\
\hline $\mathrm{CH}$ & 1 & 5000 & 4 & 52201 & MoLLIST & $\checkmark$ & Masseron et al. [139] & Empirical \\
\hline $\mathrm{NH}$ & 1 & 5000 & 1 & 12150 & MoLLIST & $\checkmark$ & Brooke et al. [140,141], Fernando et al. [142] & Empirical \\
\hline $\mathrm{OH}$ & 1 & 5000 & 2 & 54,276 & MoLLIST & $\checkmark$ & Brooke et al. [143] & Empirical \\
\hline $\mathrm{AlCl}$ & 2 & 5000 & 1 & 20245 & MoLLIST & $\checkmark$ & Yousefi and Bernath [144] & Empirical \\
\hline AlF & 1 & 5000 & 1 & 40490 & MoLLIST & $\checkmark$ & Yousefi and Bernath [144] & Empirical \\
\hline $\mathrm{OH}^{+}$ & 1 & 5000 & 2 & 12044 & MoLLIST & $\checkmark$ & Hodges and Bernath [145], Hodges et al. [146] & Empirical \\
\hline $\mathrm{CaF}$ & 1 & 5000 & 6 & 14817 & MoLLIST & $\checkmark$ & Hou and Bernath [147] & Empirical \\
\hline $\mathrm{MgF}$ & 1 & 5000 & 3 & 8136 & MoLLIST & $\checkmark$ & Hou and Bernath [148] & Empirical \\
\hline $\mathrm{KF}$ & 1 & 5000 & 2 & 10572 & MoLLIST & $\checkmark$ & Frohman et al. [149] & Empirical \\
\hline $\mathrm{NaF}$ & 1 & 5000 & 1 & 7884 & MoLLIST & $\checkmark$ & Frohman et al. [149] & Empirical \\
\hline $\mathrm{LiCl}$ & 1 & 5000 & 4 & 26260 & MoLLIST & $\checkmark$ & Bittner and Bernath [150] & Empirical \\
\hline $\mathrm{LiF}$ & 1 & 5000 & 2 & 10621 & MoLLIST & $\checkmark$ & Bittner and Bernath [150] & Empirical \\
\hline $\mathrm{MgH}$ & 2 & 5000 & 1 & 14179 & MoLLIST & $v$ & GharibNezhad et al. [151] & Empirical \\
\hline $\mathrm{TiH}$ & 1 & 5000 & 3 & 181080 & MoLLIST & $\checkmark$ & Burrows et al. [152] & Empirical \\
\hline $\mathrm{CrH}$ & 1 & 5000 & 2 & 13824 & MoLLIST & $v$ & Chowdhury et al. [153] & Empirical \\
\hline $\mathrm{FeH}$ & 1 & 5000 & 2 & 93040 & MoLLIST & $\checkmark$ & Wende et al. [154] & Empirical \\
\hline $\mathrm{HF}$ & 2 & 5000 & 1 & 7956 & Coxon-Hajig & $\checkmark$ & Coxon and Hajigeorgiou [155] & Empirical \\
\hline $\mathrm{HCl}$ & 4 & 5000 & 1 & 2588 & HITRAN & $\checkmark$ & Li et al. [156] & Empirical \\
\hline $\mathrm{CP}$ & 1 & 5000 & 2 & 28752 & MoLLIST & $v$ & Ram et al. [157] & Empirical \\
\hline $\mathrm{CN}$ & 1 & 5000 & 3 & 195120 & MoLLIST & $\checkmark$ & Brooke et al. [158] & Empirical \\
\hline $\mathrm{C}_{2}$ & 1 & 5000 & 2 & 47570 & MoLLIST & & Brooke et al. [159] & Empirical \\
\hline $\mathrm{CaH}$ & 2 & 5000 & 1 & 6000 & MoLLIST & $\boldsymbol{v}$ & Li et al. [160], Shayesteh et al. [161] & Empirical \\
\hline $\mathrm{N}_{2}$ & 1 & 10000 & $4^{a}$ & 7182000 & WCCRMT & $\checkmark$ & Western et al. [162] & Empirical \\
\hline $\mathrm{SiO}$ & 1 & 5000 & 3 & 667814 & Kurucz-SiO & & Kurucz [81] & Empirical \\
\hline $\mathrm{ScH}$ & 1 & 5000 & 6 & 1152827 & LYT & $\boldsymbol{v}$ & Lodi et al. [163] & Ab initio \\
\hline $\mathrm{LiH}$ & 1 & 12000 & 1 & 18982 & CLT & $\boldsymbol{v}$ & Coppola et al. [164] & $\mathrm{Ab}$ initio \\
\hline $\mathrm{LiH}^{+}$ & 1 & 12000 & 1 & 332 & CLT & $\checkmark$ & Coppola et al. [164] & $\mathrm{Ab}$ initio \\
\hline $\mathrm{CO}$ & 9 & 9000 & 1 & 752976 & Li2015 & $\checkmark$ & Li et al. [165] & Empirical \\
\hline $\mathrm{HeH}^{+}$ & 4 & 9000 & 1 & 1430 & ADSJAAM & $\checkmark$ & Amaral et al. [166] & $\mathrm{Ab}$ initio \\
\hline $\mathrm{HD}^{+}$ & 1 & 9000 & 1 & 10285 & ADSJAAM & $\checkmark$ & Amaral et al. [166] & Ab initio \\
\hline HD & 1 & 9000 & 1 & 5939 & ADSJAAM & $\checkmark$ & Amaral et al. [166] & $\mathrm{Ab}$ initio \\
\hline $\mathrm{CH}_{3} \mathrm{~F}$ & 1 & 300 & 1 & 139188215 & OYKYT & $\checkmark$ & Owens et al. [167] & Ab initio \\
\hline $\mathrm{AsH}_{3}$ & 1 & 300 & 1 & 3600000 & CYT18 & $v$ & Coles et al. [168] & Ab initio \\
\hline $\mathrm{P}_{2} \mathrm{H}_{2} b$ & 2 & 300 & 1 & 10667208951 & OY-Trans & $v$ & Owens and Yurchenko [169] & Ab initio \\
\hline $\mathrm{P}_{2} \mathrm{H}_{2} b$ & 2 & 300 & 1 & 11020092365 & OY-Cis & $\checkmark$ & Owens and Yurchenko [169] & $\mathrm{Ab}$ initio \\
\hline $\mathrm{PF}_{3}$ & 1 & 300 & 1 & 68000000000 & MCYTY & $\checkmark$ & Mant et al. [170] & Ab initio \\
\hline $\mathrm{CH}_{3}$ & 1 & 1500 & 1 & 2058655166 & AYYJ & $v$ & Adam et al. [171] & Ab initio \\
\hline $\mathrm{BeH}$ & 3 & 5000 & 2 & 592308 & Darby-Lewis & $\checkmark$ & Darby-Lewis et al. [172] & ExoMol \\
\hline $\mathrm{CO}_{2}$ & 13 & 4000 & 1 & 298323789 & Ames-2016 & & Huang et al. $[173,174]$ & ExoMol-like \\
\hline $\mathrm{SiH}_{2}$ & 1 & 2000 & 1 & 254061207 & CATS & $\checkmark$ & Clark et al. [175] & ExoMol \\
\hline YO & 1 & 5000 & 6 & 3520133 & SSYT & $\checkmark$ & Yurchenko et al. [176] & $\mathrm{Ab}$ initio \\
\hline
\end{tabular}

$N_{\text {iso }}$ Number of isotopologues considered; $T_{\max }$ Maximum temperature for which the line list is complete; $N_{\text {elec }}$ Number of electronic states considered; $N_{\text {lines }}$ Number of lines: value is for the main isotope. $\boldsymbol{V}$ indicates line list recommended for studies of hot atmospheres. ${ }^{a}$ The WCCRMT line list considers triplet states only. ${ }^{b}$ There are separate line lists for cis and trans $\mathrm{P}_{2} \mathrm{H}_{2}$.

Table 3

Supplementary datasets available from the website.

\begin{tabular}{llllrlll}
\hline Molecule & $N_{\text {iso }}$ & $T_{\max }$ & $N_{\text {elec }}$ & \multicolumn{1}{c}{$N_{\text {lines }}$} & DSName & Reference & Methodology \\
\hline $\mathrm{H}_{3}^{+}$ & $2^{a}$ & 4000 & 1 & 3070571 & NMT & Neale et al. [177] & ExoMol \\
$\mathrm{H}_{2} \mathrm{O}$ & $2^{b}$ & 3000 & 1 & 505806202 & BT2 & Barber et al. [178] & ExoMol \\
$\mathrm{NH}_{3}$ & $2^{c}$ & 1500 & 1 & 1138323351 & BYTe & Yurchenko et al. [179] & ExoMol \\
$\mathrm{HeH}^{+}$ & 4 & 10000 & 1 & 1431 & Engel & Engel et al. [180] & Ab initio \\
$\mathrm{HD}^{+}$ & 1 & 12000 & 1 & 10119 & CLT & Coppola et al. [164] & Ab initio \\
$\mathrm{CO}_{2}$ & 13 & 300 & 1 & 161,944 & Zak & Zak et al. [181,182,183] & ExoMol \\
$\mathrm{CO}_{2}$ & 1 & 4000 & 1 & 628324454 & CSSD-4000 & Tashkun and Perevalov [184] & Empirical \\
$\mathrm{H}_{2} \mathrm{O}$ & 1 & 300 & 1 & & WAT_UV296 & Conway et at. [185] & ExoMol. \\
\hline
\end{tabular}

$N_{\text {iso }}$ Number of isotopologues considered; $T_{\max }$ Maximum temperature for which the line list is complete; $N_{\text {elec }}$ Number of electronic states considered; $N_{\text {lines }}$ Number of lines: value is for the main isotope.

adjusted to remove transitions affected by this issue. Medvedev et al. [189] recently identified similar issues with triatomic systems but tests suggest that in practice our triatomic line lists seem largely unaffected by the problem.

Below we consider some of the line lists presented in the ExoMol database and listed in Tables 1 and 2. We restrict our discussion to issues not covered in the ExoMol2016 release or the original publications. We start by considering the 42 molecules for which line lists have been created as part of the ExoMol series as listed in Table 1.

\subsection{Diatomics}

\subsubsection{AlH, Paper XVIII}

There is a new AlHambra line list for AlH [122]. A MARVEL (measured active rotational-vibrational energy levels [190]) project was performed as part of this project meaning that many transitions are predicted with experimental accuracy. The AlHambra line list has been updated to give the uncertainty in the energy in the States file which allows users to determine the uncertainty in a given transition wavenumber. 


\subsubsection{AlO, Paper IX}

No change. The APTY line list was recently used to make a detection of AlO in an exoplanet atmosphere, the ultra-hot Jupiter exoplanet WASP-33 b [191] and hot Jupiter exoplanet WASP-43 b [192].

\subsubsection{BeH, Paper I}

The BeH "Yadin" line list was one of the first constructed by ExoMol [94]; however, the line list only included transitions within the $\mathrm{X}^{2} \Sigma^{+}$ground electronic state. Recently Darby-Lewis et al. [172] constructed line lists for BeH, BeD and BeT which consider both the ground and first excited $\left(A^{2} \Pi\right)$ states. At the same time Darby-Lewis et al. performed a MARVEL analysis and used their empirical levels in the fit. The Darby-Lewis line list is therefore more accurate and more extensive than the Yadin one which it replaces in the ExoMol database and is therefore recommended. Yadin is still available on the website though renamed to Yadin$\mathrm{BeH}$ in order to avoid conflict with the "Yadin" line lists for $\mathrm{MgH}$ and $\mathrm{CaH}$, which remain to be recommended for the IR region.

\subsection{4. $C_{2}$, Paper XXXI}

There are two new line lists for $C_{2}$ in the ExoMol2020 release: ExoMol 8state [124] and the empirical MoLLIST [159]. The MoLLIST line list only considers the much-observed Swan band while 8state covers the 8 band systems which interconnect the 8 lowest electronic states of $C_{2}$. As 8state uses empirical MARVEL energy levels [193] where available it should be as accurate as MoLLIST for the Swan band. Use of 8state is therefore recommended. Since MoLLIST line lists are recommended for other molecules and in order to avoid conflicts with the 'recommended' flag for 8 states, the $C_{2}$ MoLLIST line list is now referenced to as MoLLIST-C2 on ExoMol.

An update of the $C_{2}$ MARVEL data has just been completed [194]; this has been used to improve the 8state energies and hence transition wavenumbers. This latest version includes uncertainties in the States file.

\subsection{5. $\mathrm{CaH}$, Paper I}

The "Yadin" $\mathrm{CaH}$ line list only considers transitions within the $X^{2} \Sigma^{+}$ground electronic state [94]. MoLLIST provides a rovibronic line list for the $X^{2} \Sigma^{+}-X^{2} \Sigma^{+}, A^{2} \Pi-X^{2} \Sigma^{+}, B^{2} \Sigma^{+}-X^{2} \Sigma^{+}$and $\mathrm{E}^{2} \Pi-\mathrm{X}^{2} \Sigma^{+}$systems due to Li et al. [160], Shayesteh et al. [161], Ram et al. [195]. Both line lists are included in the ExoMol database; work is in progress on creating a single unified line list which will extend the range of rovibronic transitions considered. In the meantime both line lists are recommended.

\subsubsection{CaO, Paper XIII}

No change.

\subsubsection{CS, Paper XII}

No change. We note that a line list for CS has also recently been supplied by Hou and Wei [196] and Xing et al. [197] have extended consideration to rovibronic transitions for the lowest, $A^{1} \Pi$ - X ${ }^{1} \Sigma^{+}$, allowed electronic band.

\subsection{8. $\mathrm{KCl}$, Paper $V$ \\ No change}

\subsection{9. $\mathrm{MgH}$, Paper I}

The ExoMol "Yadin" line list for MgH only considers transitions within the $X^{2} \Sigma^{+}$ground electronic state [94]. MoLLIST provides a rovibronic line list containing $\mathrm{A}^{2} \Pi-\mathrm{X}^{2} \Sigma^{+}$and $\mathrm{B}^{\prime}{ }^{2} \Sigma^{+}-\mathrm{X}^{2} \Sigma^{+}$ transitions due to GharibNezhad et al. [151]. Both line lists are included in the ExoMol database; work is in progress on creating a single unified line list which will extend the range of rovibronic transitions considered. In the meantime both line lists are recommended.

\subsubsection{MgO, Paper XXXII}

There is a new LiTY line list for MgO [125].

\subsubsection{1. $\mathrm{NaCl}$, Paper $\mathrm{V}$}

No change.

\subsubsection{2. $\mathrm{NaH}$, Paper $\mathrm{X}$}

No change.

\subsubsection{NO, Paper XXI}

There is a new NOName line list for NO [115]. This line list was constructed using a combination of standard ExoMol and empirical methodologies, and also included a MARVEL project. Its transition frequencies should therefore be close to experimental accuracy. NOName has largely been adopted for the new release of HITEMP [77]

NOName only covers transitions between levels which lie within the $\mathrm{X}^{2} \Pi$ ground electronic state of NO. A new line list which includes ultraviolet rovibronic transitions of NO is currently being constructed.

\subsubsection{PH, Paper XXXIV}

There is a new LaTY line list for PH [127].

\subsubsection{PN, Paper VI}

No change. An extended line list covering visible and ultra violet (UV), accompanied by a MARVEL project is currently in progress.

\subsubsection{PO, Paper XXIII}

There is a new POPS line list for PO [117].

\subsubsection{PS, Paper XXIII}

There is a new POPS line list for PS [117].

\subsubsection{SH, Paper XXXVI}

There are two new ExoMol line lists for SH: SNaSH [120] which only covers transitions within the $\mathrm{X}^{2} \Pi$ ground electronic state of SH and the newer GYT line list of Gorman et al. [129]. GYT considers both transitions within $\mathrm{X}^{2} \Pi$ and vibronic transitions in the $\mathrm{A}^{2} \Sigma^{+}-\mathrm{X}^{2} \Pi$ band system. As GYT also improves the accuracy of the $\mathrm{X}$ state transitions, it is recommended for all applications. The $\mathrm{SNaSH}$ SH line list is also renamed to $\mathrm{SNaSH}-\mathrm{SH}$ in order to avoid a conflict and retained only for completeness.

\subsubsection{SiO, Paper II}

The ExoMol SiO line lists only consider transitions within the $\mathrm{X}^{1} \Sigma^{+}$ground electronic state [95]; these line lists have been widely used including a recent determination of isotopologue ratios in Arcturus [198]. For this release of ExoMol, an empirical and less accurate SiO line list by Kurucz [81] covering the X-X, A-X and $\mathrm{E}-\mathrm{X}$ electronic bands was added. Given the importance of $\mathrm{SiO}$ for lava planets [65], construction of a comprehensive (accurate and complete) rovibronic line list for SiO covering both IR and ultraviolet would be useful.

\subsubsection{SiH, Paper XXIV}

There is a new SiGHTLY line list for $\mathrm{SiH}$ [118].

\subsubsection{SiS, Paper XXV}

There is a new UCTY line list for SiS [119].

\subsubsection{SN, Paper XXVI}

There is a new SNaSH line list for SN [120]. 


\subsubsection{TiO, Paper XXXIII}

There is a new Toto line list for TiO [126]. The line list for the main isotopologue $\left({ }^{48} \mathrm{Ti}^{16} \mathrm{O}\right)$ used empirical energies from an associated MARVEL study [199] while the energy levels of other isotopologues were improved using the procedure of Polyansky et al. [113]. The Toto line list has been updated to give MARVEL uncertainties, where available, in the States file. For other states the uncertainties were estimated as follows: $1 \mathrm{~cm}^{-1}$ for all levels in vibronic states that contain levels determined by MARVEL, $10 \mathrm{~cm}^{-1}$ for levels in those electronic states whose excitation energy $\left(T_{e} / T_{0}\right)$ is known from experiment and $100 \mathrm{~cm}^{-1}$ for all other levels.

TiO is a particularly important species in the atmosphere of cool stars and has been detected in exoplanets [200,201]. The Toto line list is significantly better at reproducing stellar spectra than the line lists due to Plez [202] and Schwenke [203] which it supersedes. Furthermore a recent study of $\mathrm{Ti}$ isotope abundances in two M-dwarf stars demonstrated the accuracy of the line lists for several isotopologues of $\mathrm{TiO}$ [42]. However, TiO remains a challenging system to treat using $a b$ initio electronic structure methods (see McKemmish et al. [204]) and further work is required before there is a definitive line list for TiO.

\subsubsection{VO, Paper XVIII}

There is a new VOMYT line list for VO [112] which has been used to tentatively identify $\mathrm{VO}$ in the atmosphere of exoplanets $[205,206]$. We note that VO spectra display particularly large splittings due to hyperfine effects as well as transitions which are only allowed due to these effects [207]. VOMYT does not include hyperfine effects in its spectroscopic model meaning that the line list is unsuitable for high resolution studies. We plan to address this problem by both performing a hyperfine resolved MARVEL study and extending Duo to allow for hyperfine effects.

\subsection{Triatomics}

\subsection{1. $\mathrm{CO}_{2}$, Paper XXXIX}

Line lists for hot $\mathrm{CO}_{2}$ have been constructed by NASA Ames $[173,173]$ using methodologies similar to those employed by ExoMol. The CDSD-4000 hot line list due to Tashkun and Perevalov [184] and the room temperature line lists due to Zak et al. [181-183] are also available on the ExoMol website. The CDSD4000 line list is based on the use of an effective Hamiltonian which tends to miss contributions from unobserved hot bands.

A new ExoMol line list UCL-4000 for $\mathrm{CO}_{2}$ has been produced [132] using an accurate $a b$ initio dipole moment surface (DMS) by Polyansky et al. [208] and empirical potential energy surface (PES) Ames-2016 by Huang et al. [209]; where possible computed energies have been replaced by empirical ones derived from $\mathrm{HI}-$ TRAN. The UCL-4000 line list covers the wavenumber range 0$20000 \mathrm{~cm}^{-1}$ and should be applicable for temperatures up to $4000 \mathrm{~K}[132]$. It is recommended for use in high temperature applications.

\subsection{2. $\mathrm{HCN} / \mathrm{HNC}$, Paper III}

The combined $\mathrm{HCN} / \mathrm{HNC}$ ExoMol line list of Barber et al [96] made extensive use of empirical energy levels due to Mellau $[210,211]$. This has enabled the line list to be successfully used in high-resolution Doppler spectroscopy studies of exoplanets [212214].

\subsection{3. $\mathrm{H}_{2} \mathrm{O}$, Papers $\mathrm{XIX}$ and $\mathrm{XXX}$}

Water is one of the most widely studied molecules and its spectrum has been detected in a variety of exoplanets, in many cases as the only clearly identifiable molecule [206,215]. Good water line lists have been available for some time, in particular the Ames line list of Partridge and Schwenke [216] and the BT2 line list due to Barber et al. [178]. While BT2 was significantly more complete than Ames, the Ames line list was more accurate, particularly at infrared wavelengths. A new line list, known as POKAZATEL [61] has been generated by the ExoMol project. POKAZATEL is complete in the sense that it contains transitions between all bound rotation-vibration states in the molecule and thus is reliable for temperatures above $3000 \mathrm{~K}$ where the earlier line lists are not valid. POKAZATEL is also intrinsically more accurate than the Ames line list and, indeed, as many of its energy levels have been replaced by empirical energy levels generated using MARVEL [217], key transition frequencies are actually reproduced to experimental accuracy. POKAZATEL should therefore be used in preference to the earlier line lists.

It has become apparent that the rotation-vibration spectrum of water is responsible for weak but observable near-UV absorption in the Earth's atmosphere [218]. The POKAZATEL line list, which used the $a b$ initio LTP2001S DMS of Lodi et al. [219], appears to underestimate the strength of water absorption in the blue and near-UV. As a response to this Conway and co-workers have recently developed a more accurate global dipole moment surface [220]. Through a significant number of comparisons against high quality experimental and theoretical sources of spectroscopic data [221,222], their results suggest there may be advantages of using their line lists in particular regions, primarily at short wavelengths. For this reason, a new room temperature $\mathrm{H}_{2}^{16} \mathrm{O}$ line list called WAT_UV296 [185] has been computed. This line list is available on the website and should be used for room temperature studies of spectra with $\lambda<0.5 \mu \mathrm{m}$. A high temperature companion to this line list will form the basis of the updated HITEMP database [79].

Polyansky et al. [113] provided the HotWat78 line lists for $\mathrm{H}_{2}{ }^{17} \mathrm{O}$ and $\mathrm{H}_{2}^{18} \mathrm{O}$. The energy levels of this line list were improved using MARVEL energies [223,224]. Furthermore, Polyansky et al. [113] developed a method which gives excellent isotopologue energy levels for states only observed for $\mathrm{H}_{2}{ }^{16} \mathrm{O}$. Finally the VTT line list due to Voronin et al. [136] remains the recommended one for HDO; a new, improved HDO line list is currently under construction [225].

A new room temperature line list CKYKKY for $\mathrm{H}_{2} \mathrm{O}$ containing electric quadrupole moments was computed using an $a b$ initio quadrupole moment surface [226] and accurate empirical PES by Mizus et al. [227] with the variational program TROVE [228]. The energies were replaced by the $\mathrm{H}_{2} \mathrm{O}$ IUPAC MARVEL values [217] or HITRAN values [229], if available.

\subsection{4. $\mathrm{H}_{2} \mathrm{~S}$, Paper XVI}

There is a new ExoMol AYT2 line list for hydrogen sulphide due to Azzam et al. [110]. Since completion of this line list a MARVEL project has been performed for $\mathrm{H}_{2} \mathrm{~S}$ [230]; the AYT2 line list is being updated to use these improved energy levels.

\subsection{5. $\mathrm{H}_{3}^{+}$, Paper XX}

The new ExoMol MiZATeP line list of Mizus et al. [114] replaces that of Neale et al. [177] (NMT). The new line list uses empirical energy levels from the MARVEL study of Furtenbacher et al. [231] Astronomy makes wide use of $\mathrm{H}_{3}^{+}$line lists and is reliant on $a b$ initio line intensities since no absolute line intensities have been measured in the laboratory, see Petrignani et al. [232]. This astronomical work on $\mathrm{H}_{3}^{+}$has recently been reviewed by Miller et al. [233]. The ST $\mathrm{H}_{2} \mathrm{D}^{+}$line list due to Sochi and Tennyson [135] plus the newly generated $\mathrm{D}_{3}^{+}$and $\mathrm{D}_{2} \mathrm{H}^{+}$line lists will be MARVELised and released in the near future.

\subsection{6. $\mathrm{SO}_{2}$, Paper XVII}

The ExoAmes line list for $\mathrm{SO}_{2}[193]$ is unchanged since the ExoMol2016 release. However, a MARVEL set of energy levels for $\mathrm{SO}_{2}$ are now available [234] and will be used to update ExoAmes in the near future. 


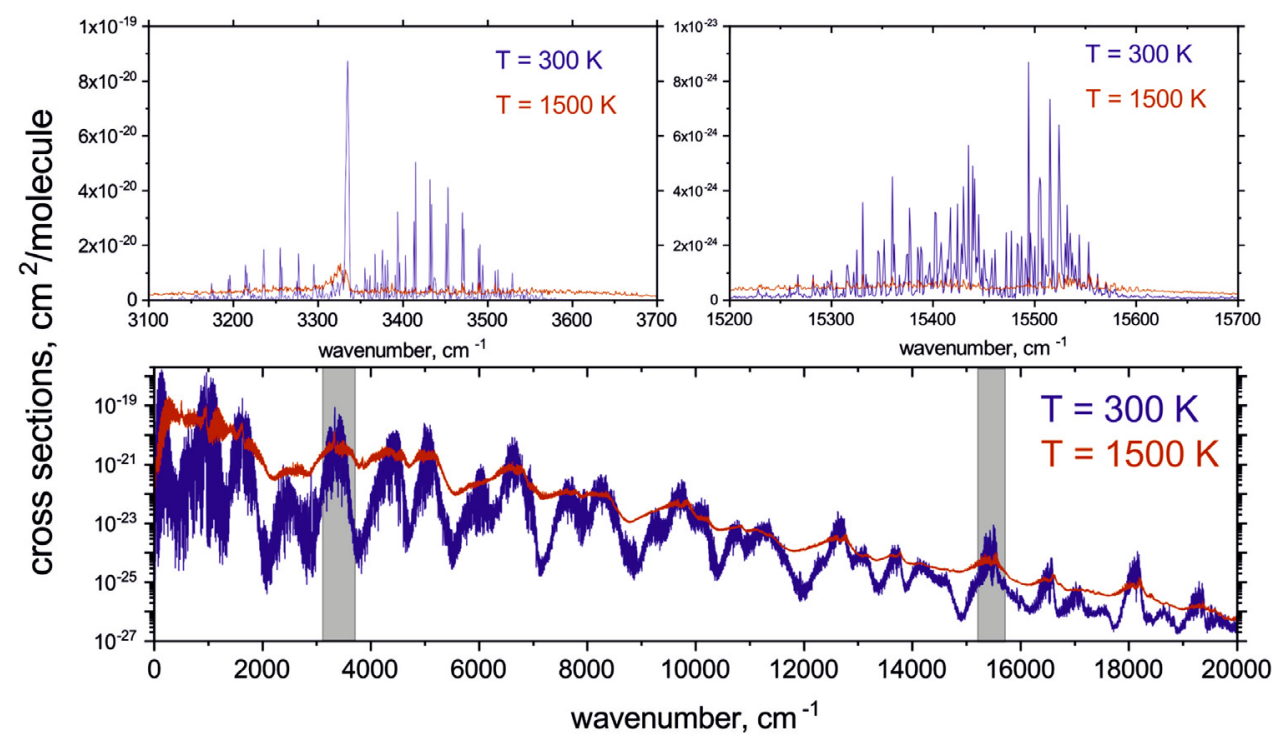

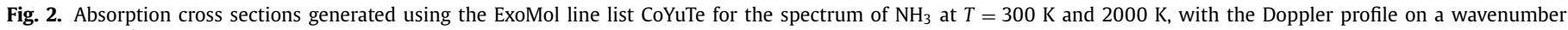
grid of $1 \mathrm{~cm}^{-1}$. The cross sections were computed using the ExoCross program, which is developed to work with two file line list structure adopted by ExoMol.

\subsection{Tetratomics}

\subsubsection{HCCH, Paper XXXVII}

The new aCeTY line list for acetylene due to Chubb et al. [130] has recently been released. This line list already incorporated the MARVEL energies of Chubb et al. [235] and gives uncertainties in the energy levels as part of the States file.

\subsection{2. $\mathrm{H}_{2} \mathrm{CO}$, Paper VIII}

The AYTY formaldehyde line list [102] is in the process of being updated with empirical energy levels produced by a recent MARVEL study [236]; this will make the line list suitable for high resolution studies.

\subsection{3. $\mathrm{H}_{2} \mathrm{O}_{2}$, Paper $\mathrm{XV}$}

There is a new ExoMol APTY line list for hydrogen peroxide [237]. We note because of difficulties with assigning spectra, even at room temperature HITRAN is very incomplete for $\mathrm{H}_{2} \mathrm{O}_{2}$. APTY should give complete coverage at infrared wavelengths. Recently APTY was used to suggest the importance of $\mathrm{H}_{2} \mathrm{O}_{2}$ as a greenhouse gas on oxidised Early Mars [238].

\subsection{4. $\mathrm{H}_{3} \mathrm{O}^{+}$, Paper $\mathrm{XL}$}

A line list for the hydronium ion, $\mathrm{H}_{3} \mathrm{O}^{+}$, has recently been constructed [133] in response to a laboratory study by Bourgalais et al. [239] which suggested both that hydronium is likely to be a dominant molecular ion in gaseous exoplanets and that it should be detectable by upcoming space missions.

\subsection{5. $\mathrm{NH}_{3}$, Paper XXXV}

A new ExoMol line list for ammonia called CoYuTe [128] has recently been completed. CoYuTe, which also uses MARVEL energy levels [188], is both more accurate and more extensive than the BYTe line list [179] it replaces. In particular, CoYuTe was found to provide a good model of the Jovian optical absorption spectrum due to ammonia [34] although with a shift in positions of the main bands. More laboratory work or analysis of existing laboratory work on the visible spectrum of ammonia will be required to resolve this issue. An illustration of the ammonia absorption cross sections computed using CoYuTe is given in Fig. 2.

\subsection{6. $\mathrm{PH}_{3}$, Paper VII}

No change. Sousa-Silva et al. [240] use the SAITY $\mathrm{PH}_{3}$ line list to assess the detectability of a possible $\mathrm{PH}_{3}$ biosignature. A MARVEL project on the $\mathrm{PH}_{3}$ experimental data is under way, which will improve the quality of the line positions in SAITY.

\subsection{7. $\mathrm{SO}_{3}$, Paper XVII}

There is a new UYT2 line list for $\mathrm{SO}_{3}$ [111].

\subsection{Pentatomics}

\subsection{1. $\mathrm{CH}_{3} \mathrm{Cl}$, Paper XXIX}

There are new OYT line lists for methyl chloride [123] covering both major isotopes of chlorine.

\subsection{2. $\mathrm{CH}_{4}$, Paper IV}

The ExoMol YT10to10 line list [97] has been extended to higher temperatures [98] to give the YT34to10 line list. However, at present the TheoReTS line list of Rey et al. [241] is the most accurate methane line list available and is recommended for detailed studies. This line list forms the basis of the very recent update to HITEMP [78].

The importance of methane and the huge number of lines required to accurately represent the opacity of hot methane [31] has led to this system being the primary focus of studies aimed at compacting these lines into a more manageable form [98,242,243]. This issue is discussed in Section 5.3.2.

\subsection{3. $\mathrm{HNO}_{3}$, Paper $\mathrm{XI}$}

No change. We note that, like $\mathrm{H}_{2} \mathrm{O}_{2}$, because of difficulties with assigning spectra even at room temperature, HITRAN is very incomplete for nitric acid. The AlJS line list gives complete coverage at infrared wavelengths.

\subsection{4. $\mathrm{SiH}_{4}$, Paper XXII}

There is a new OY2T line list for silane [116].

\subsection{Larger molecules}

\subsection{1. $\mathrm{C}_{2} \mathrm{H}_{4}$, Paper XXVII}

There is a new MaYTY line list for ethylene [121]. 


\subsection{MoLLIST species}

A number of diatomic species have line lists provided by the MoLLIST website [82]. These have been incorporated into the ExoMol database, which now includes empirically-derived line lists for the following species [83]: $\mathrm{CH}$ [139]; $\mathrm{NH}$ [140-142]; OH [143]; $\mathrm{AlCl}$ [144]; AlF [144]; $\mathrm{OH}^{+}$[145,146];CaF [147]; MgF [148]; KF and $\mathrm{NaF}$ [149]; $\mathrm{LiCl}$ and $\mathrm{LiF}$ [150]; $\mathrm{MgH}$ [151]; $\mathrm{TiH}$ [152]; $\mathrm{CrH}$ [153]; $\mathrm{FeH}$ [154]; CP [157]; CN [158]; and CaH [160,161]. See Table 2 for further details.

\subsection{HITRAN}

In general the line lists contained in the HITRAN database [229] do not provide the temperature coverage needed for astrophysical applications. However, for a few diatomic species very extensive line lists have been constructed. The line lists presented by Li et al. [156] for $\mathrm{HCl}$ and $\mathrm{Li}$ et al. [165] for CO are both valid over an extended temperature range and have been included in the ExoMol database.

The HF line list from Li et al. [244] and Coxon and Hajigeorgiou [155] is also adopted directly from HITRAN.

\section{8. $\mathrm{N}_{2}$}

The empirical WCCRMT nitrogen molecule line list of Western et al. [162] is somewhat unusual in that it only considers transitions between excited, triplet states of the molecule. However, $\mathrm{N}_{2}$ is an important molecule with very limited spectral signature at long wavelengths and WCCRMT may prove useful in hot or irradiated, usually non-LTE environments. For the partition function of $\mathrm{N}_{2}$ the ground electronic state data from TIPS [245] were used.

\section{9. $\mathrm{HD}, \mathrm{HD}^{+}, \mathrm{HeH}^{+}$}

One and two electron diatomics are important primordial species and may well play a role elsewhere. Recently Amaral et al. [166] created new line lists using high level $a b$ initio procedures. These line lists have been included in the database with the name ADSJAAM. In particular the ADSJAAM line lists for $\mathrm{HeH}^{+}$and $\mathrm{HD}$ supersedes those due to Engel et al. [180] and Coppola et al. [164], respectively.

3.10. $\mathrm{H}_{2}$

A new external line list RACPPK by Roueff et al. [138] for the ground electronic state of $\mathrm{H}_{2}$ was converted to the ExoMol format. The line list contains a combination of electric quadrupole and magnetic dipole transitions computed from first principles.

\subsection{Other $\mathrm{ab}$ initio line lists}

A number of other $a b$ initio line lists are also available. In general $a b$ initio line lists are only accurate for systems with very few electrons. The CLT line lists for $\mathrm{LiH}$ and $\mathrm{LiH}^{+}$due to Coppola et al. [164] fall into the few-electron category and should be reliable. The other $a b$ initio line lists provided, namely those for ScH [163], $\mathrm{CH}_{3} \mathrm{~F}$ [167], $\mathrm{AsH}_{3}$ [168], $\mathrm{P}_{2} \mathrm{H}_{2}$ [169], $\mathrm{PF}_{3}$ [170], $\mathrm{CH}_{3}$ [171] and $\mathrm{YO}$ [176] must be regarded as intrinsically less accurate than other line lists provided by the database. In the case of YO, work is in progress aimed at producing an empirical line list based on available experimental data. $\mathrm{P}_{2} \mathrm{H}_{2}$ occurs as two distinct isomers, the cis and trans forms. Separate line lists are provided for cis- $\mathrm{P}_{2} \mathrm{H}_{2}$ and trans $-\mathrm{P}_{2} \mathrm{H}_{2}$.

\subsection{2. $\mathrm{SiH}_{2}$}

A new line list for $\mathrm{SiH}_{2}$ [175] has been recently constructed in its ground electronic state using the standard ExoMol technology [93]. The set of experimental data or their quality were, however, very limited which may affect the quality of the hot bands, which basically has to rely on the quality of the $a b$ initio PES.

\subsection{3. $\mathrm{NiH}$}

Empirical line lists for three isotopologues of $\mathrm{NiH}\left({ }^{58} \mathrm{NiH},{ }^{60} \mathrm{NiH}\right.$, $\left.{ }^{62} \mathrm{NiH}\right)$ known as HRV. These line lists were constructed by Vallon et al. [246] and Harker et al. [247]. However, at present these line lists are not available in ExoMol format or accessible through the API.

\subsection{Partition functions}

The temperature dependent partition functions for most of the ExoMol line lists are computed using the corresponding energy levels as collected in the States files as

$Q(T)=\sum_{i} g_{\text {ns }}^{(i)} J_{i}\left(J_{i}+1\right) \exp \left(-\frac{c_{2} \tilde{E}_{i}}{T}\right)$,

where $g_{n s}^{(i)}$ is the state dependent nuclear statistical weight, $J_{i}$ is the total angular momentum of the state $i, c_{2}$ is the second radiation constant $(\mathrm{K} \mathrm{cm})$ and $\tilde{E}_{i}$ is the corresponding energy term value $\left(\mathrm{cm}^{-1}\right)$. ExoMol uses the HITRAN convention [248], where the entire, integer $g_{i}=g_{\text {ns }} J_{i}\left(J_{i}+1\right)$ factors are explicitly included.

Some of the line lists in the ExoMol database do not provide sufficiently complete sets states for a proper evaluation of the molecular partition functions. For example, most of the MoLLIST line lists are constructed from or based on the measured data only and therefore can be severely incomplete. In these cases the partition functions are either taken from external sources, such as the TIPS database [245], generated using the empirical expansions, such as by Irwin [249], Sauval and Tatum [250], Irwin [251], Barklem and Collet [252] or extrapolated using simplified models [253]. The external partition functions often include the $g_{\text {ns }}^{(i)}$ factors in the astrophysical convention (see, e.g. Pavlenko et al. [198]) and therefore have to be transformed to the HITRAN convention.

We strongly recommend that users use these partition functions rather than attempting to compute their own using the ExoMol States files. These States files are not constructed with a view to delivering reliable partition functions and in a number of cases use of them has been found to lead to problems.

\subsection{VUV Sections}

A section is provided based on measured ultraviolet cross sections for key species. For many molecules their ultraviolet spectrum is a mixture of line and quasi-continuum absorption which cannot be represented as a line list. The new VUV section will provide temperature-dependent absorption cross sections. Recent results suggest the VUV absorption by $\mathrm{H}_{2} \mathrm{O}$ enhances the production of $\mathrm{OH}$, which plays an important role in the atmospheric chemistry of exoplanetary atmospheres [254]. Currently, the VUV cross sections are provided for $\mathrm{H}_{2} \mathrm{O}, \mathrm{H}_{2}, \mathrm{CO}_{2}, \mathrm{SO}_{2}, \mathrm{NH}_{3}, \mathrm{H}_{2} \mathrm{CO}$ and $\mathrm{C}_{2} \mathrm{H}_{4}$ measured by Fateev's lab at the Danish Technical University (DTU) [255] and for $\mathrm{CO}_{2}$ by Venot et al. [256]. The temperature and wavelength coverage is illustrated in Table 4.

The cross sections are given in the common two-column ASCII format separated by spaces, where the first column contains the wavelength in $\mathrm{nm}$ and the second column contains the absorption cross sections in $\mathrm{cm}^{2} /$ molecule using Fortran format: 
Table 4

VUV absorption cross sections on ExoMol, grid spacing $0.01-0.015 \mathrm{~nm}$, presented in natural abundance.

\begin{tabular}{lll}
\hline $\begin{array}{l}\text { Molecule } \\
\text { DTU data }\end{array}$ & Temperature $(\mathrm{K})$ & Range $(\mathrm{nm})$ \\
\hline $\mathrm{H}_{2} \mathrm{O}$ & 423 & \\
$\mathrm{H}_{2} \mathrm{O}$ & 573 & $110-230$ \\
$\mathrm{H}_{2} \mathrm{O}$ & 1630 & $110-230$ \\
$\mathrm{H}_{2} \mathrm{O}$ & 1773 & $182-237$ \\
$\mathrm{CO}_{2}$ & 1160 & $182-237$ \\
$\mathrm{SO}_{2}$ & 423 & $109-324$ \\
$\mathrm{NH}_{3}$ & 289 & $110-230$ \\
$\mathrm{NH}_{3}$ & 296 & $113-201$ \\
$\mathrm{H}_{2} \mathrm{CO}$ & 303 & $113-201$ \\
$\mathrm{H}_{2} \mathrm{CO}$ & 353 & $110-230$ \\
$\mathrm{H}_{2} \mathrm{CO}$ & 423 & $110-230$ \\
$\mathrm{H}_{2} \mathrm{CO}$ & 573 & $110-230$ \\
$\mathrm{C}_{2} \mathrm{H}_{4}$ & 562 & $110-230$ \\
$\mathrm{CO}_{2}$ & Venot et al. [256] & $113-201$ \\
\hline
\end{tabular}

Table 5

Summary of data provided by the ExoMol Database .

\begin{tabular}{l}
\hline Data type \\
\hline Line lists \\
Absorption cross sections \\
VUV absorption cross sections \\
Pressure broadening coefficients \\
Temperature dependent super-lines (histograms) \\
Partition functions \\
Cooling functions \\
Specific heat - heat capacity \\
Examples of ExoCross input files \\
Temperature and pressure dependent opacities \\
Spectroscopic Models
\end{tabular}

$(\mathrm{F} 10.3,1 \mathrm{x}, \mathrm{E} 13.6)$. The VUV cross section file names have the following structure

$$
1<\text { ISOTOPOLOGUE }>_{-}<\text {DATASET }>_{-}<\text {RANGE }>{ }_{-} \text {T }
$$

$<$ TEMP $>K_{-}$P $<$PRESSURE $>$bar _- $<$STEP $>$.nm ', where ISOTOPOLOGUE is the isotopologue name, DATASET is the name of the line list, RANGE is the wavelength range in nm, TEMP is the temperature in K, PRESSURE is the pressure in bar, STEP is the wavelength step in $\mathrm{nm}$. For example, the States file of the VUV line list for $\mathrm{CO}_{2}$ has the filename:

12C-1202_Venot-2018_116.90-230.00_T0800K_P0bar_ 0.03.nm.

\section{Data provided}

Table 5 provides a summary of different types of data provided. The website provides two routes to accessing these data. Users can search by molecule which will show all the types of data available for each isotopologue. Alternatively it is possible to search by data type which generates a list of molecules for which data of the specified type is available. The following section lists the data types and their file name extensions for the various data and metadata provided by ExoMol. A more technical specification of the data structures and how to access data using the application program interface (API) is provided in Section 5.

\subsection{Data structure}

The general ExoMol approach is molecule-by-molecule: a comprehensive line list is created for a particular molecule and made available in the database. The line list for each isotopologue is stored as a separate data structure which can be accessed directly or via the application program interface (API) described in the following sections. Table 6 specifies the file types that can be available for each isotopologue. The . states and .trans files are the heart of the ExoMol data structure [257] and define what has become known as the "ExoMol format". These files are available for all isotopologues; other files may not be. ExoMol format files can be used and provided by the effective Hamiltonian code PGOPHER [258].

A manual providing technical specifications of the database is included as supplementary data to this article and is included on the website where it will be updated as the data base evolves. Only the relatively few changes that have been implemented since the 2016 release are discussed below.

\subsection{Super-lines}

Super-lines $[80,98]$ represent a novel, compact way of storing the opacity data. Super-lines are constructed as temperaturedependent intensity histograms by summing all absorption coefficients within a wavenumber bin centred around a grid point $\tilde{v}_{k}$. For each $\tilde{v}_{k}$ the total absorption intensity $I_{k}(T)$ is computed as a sum of absorption line intensities $I_{i f}$ from all $i \rightarrow f$ transitions falling into the wavenumber bin $\left[\tilde{v}_{k}-\Delta \tilde{v}_{k} / 2 \ldots \tilde{v}_{k}+\Delta \tilde{v}_{k} / 2\right]$ at the given temperature $T$. Each grid point $\tilde{v}_{k}$ is then treated as a line position of an artificial transition (super-line) with an effective absorption intensity $I_{k}(T)$. The number of data points in the superlines can be drastically reduced without significant loss of accuracy when computing pressure-dependent cross sections and can be combined with standard, pressure, temperature and frequency dependent line profiles. The super-lines cannot be associated with any specific upper/lower states and therefore the line broadening parameters used cannot depend on quantum numbers.

The super-line list files have the format of cross sections represented by two columns, wavenumbers $\left(\mathrm{cm}^{-1}\right)$ and superline absorption intensity ( $\mathrm{cm} /$ molecule). The Fortran format is (F12.6,1x,ES14.8). The super-lines are computed on a grid of temperatures from $100 \mathrm{~K}$ to $T_{\max }$. For the highest resolution an adaptive grid of $R=1000000$ is used. For the technical details of super-line construction and numerical tests see Yurchenko et al. [98].

Super-lines are currently available for $\mathrm{H}_{2} \mathrm{O}, \mathrm{NH}_{3}, \mathrm{CH}_{3} \mathrm{Cl}, \mathrm{C}_{2} \mathrm{H}_{2}$ and $\mathrm{C}_{2} \mathrm{H}_{4}$.

\subsection{Specific heat}

The specific heat at constant pressure $C_{p}$ is computed on a grid of temperatures of $1 \mathrm{~K}$ from the molecule partition function as given by [259]

$C_{p}(T)=R\left[\frac{Q^{\prime \prime}}{Q}-\left(\frac{Q^{\prime}}{Q}\right)^{2}\right]+\frac{5 R}{2}$,

where the second term is the translational contribution,

$Q^{\prime}(T)=T \frac{d Q}{d T}$,

$Q^{\prime \prime}(T)=T^{2} \frac{d^{2} Q}{d T^{2}}+2 Q^{\prime}$

and $R$ is the gas constant. The specific heat values are given in units of $\mathrm{J} \mathrm{mol}^{-1} \mathrm{~K}^{-1}$ and are currently provided for $\mathrm{H}_{2} \mathrm{O}$ (taken from Furtenbacher et al. [259]) and $\mathrm{CH}_{4}$ (computed using the YT10to10 ro-vibrational energies [97]) only (see Fig. 3).

\subsection{Line shifts using the diet format}

For high resolution applications it will be important to take into account the pressure dependent line shifts. To this end a line 
Table 6

Specification of the ExoMol file types. (Contents in brackets are optional).

\begin{tabular}{llll}
\hline File extension & $N_{\text {files }}$ & File DSname & Contents \\
\hline .all & 1 & Master & Single file defining contents of the ExoMol database. \\
.def & $N_{\text {tot }}$ & Definition & Defines contents of other files for each isotopologue. \\
. states & $N_{\text {tot }}$ & States & Energy levels, quantum numbers, lifetimes, (Landé g-factors, Uncertainties). \\
. trans & $a$ & Transitions & Einstein A coefficients, (wavenumber). \\
. broad & $N_{\text {mol }}$ & Broadening & Parameters for pressure-dependent line profiles. \\
. cross & $b$ & Cross sections & Temperature or temperature and pressure-dependent cross sections. \\
. kcoef & $c$ & $k$-coefficients & Temperature and pressure-dependent $k$-coefficients. \\
.pf & $N_{\text {tot }}$ & Partition function & Temperature-dependent partition function, (cooling function). \\
.dipoles & $N_{\text {tot }}$ & Dipoles & Transition dipoles including phases. \\
. super & $d$ & Super-lines & Temperature dependent super-lines ((histograms) on a wavenumber grid. \\
.nm & $e$ & VUV cross sections & Temperature and pressure dependent VUV cross-sections (wavelength, nm). \\
.fits, .h5, .kta & $f$ & Opacities & Temperature and pressure dependent opacitities for radiative-transfer applications. \\
. overview & $N_{\text {mol }}$ & Overview & Overview of datasets available. \\
.readme & $N_{\text {iso }}$ & Readme & Specifies data formats. \\
.model & $N_{\text {iso }}$ & Model & Model specification. \\
\hline
\end{tabular}

$N_{\text {files }}$ total number of possible files; $N_{\text {mol }}$ Number of molecules in the database; $N_{\text {tot }}$ is the sum of $N_{\text {iso }}$ for the $N_{\text {mol }}$ molecules in the database; $N_{\text {iso }}$ Number of isotopologues considered for the given molecule. ${ }^{a}$ There are $N_{\text {tot }}$ sets of .trans files but for molecules with large numbers of transitions the .trans files are subdivided into wavenumber regions. ${ }^{b}$ There are $N_{\text {cross }}$ sets of . cross files for isotopologue. ${ }^{c}$ There are $N_{\text {kcoef }}$ sets of . kcoef files for each isotopoplogue. ${ }^{d}$ There are $N_{T}$ sets of $T$-dependent super-lines. ${ }^{e}$ There are $N_{V U V}$ sets of VUV cross sections. ${ }^{f}$ Set of opacity files in the format native to specific radiative-transfer programs.

Table 7

File $1 \mathrm{H}-1602$ _- aO . shiftA pressure line . shift file for $\mathrm{H}_{2} \mathrm{O}$ : portion of the file (upper part); field specification (lower part).

\begin{tabular}{|c|c|c|c|}
\hline Code & Shift & $\mathrm{J}$ & \\
\hline $\mathrm{a} 0$ & 0.0001 & 0 & \\
\hline $\mathrm{a} 0$ & 0.0001 & 1 & \\
\hline $\mathrm{a} 0$ & 0.0001 & 2 & \\
\hline $\mathrm{a} 0$ & 0.0001 & 3 & \\
\hline $\mathrm{a} 0$ & 0.0001 & 4 & \\
\hline Field & Fortran Format & C Format & Description \\
\hline code & A2 & $\% 2 \mathrm{~s}$ & Code identifying quantum number set following $\mathrm{J}^{\prime \prime *}$ \\
\hline$\gamma_{\text {ref }}$ & F15.6 & $\% 15.6 f$ & Line shift at reference temperature and pressure in $\mathrm{cm}^{-1}$ \\
\hline$J^{\prime \prime}$ & I7/F7.1 & $\% 7 d$ & Lower $J$-quantum number \\
\hline
\end{tabular}

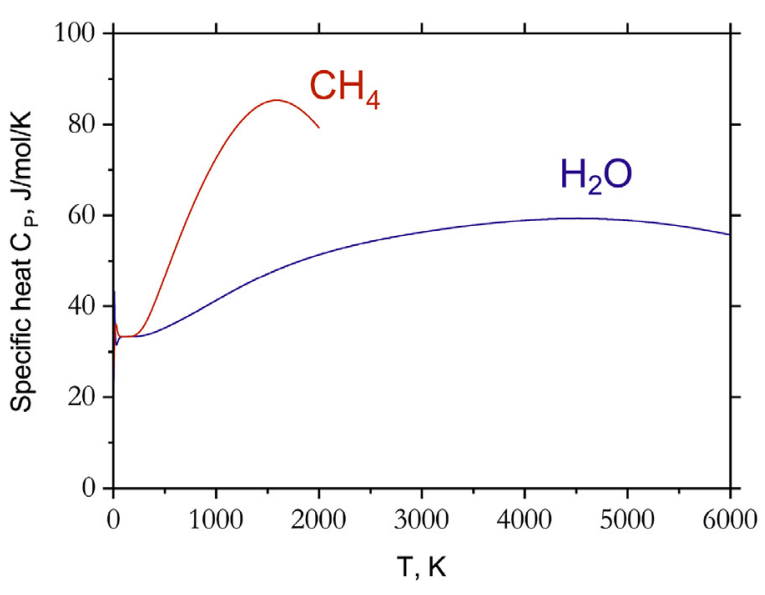

Fig. 3. Specific heat at constant pressure of $\mathrm{H}_{2} \mathrm{O}$ as computed by Furtenbacher et al. [259] and $\mathrm{CH}_{4}$ generated the YT10to10 ro-vibrational energies [97].

shift diet has been introduced using the same data structure used for pressure dependent line broadening diet [260]. An example for $\mathrm{H}_{2} \mathrm{O}$ is given in Table 7 .

\subsection{New broadening parameters}

The pressure broadening parameters are provided for 15 molecules and are listed in Table 8 using the pressure-broadening diet [260].
Table 8

Pressure broadening parameters in ExoMol.

\begin{tabular}{lll}
\hline Molecule & Broadener & Scheme \\
\hline $\mathrm{CS}$ & air, self & $\mathrm{a} 0$ \\
$\mathrm{HCl}$ & $\mathrm{H}_{2}, \mathrm{He}$, self, air, $\mathrm{CO}_{2}$ & $\mathrm{a} 0$ \\
$\mathrm{HF}$ & $\mathrm{H}_{2}, \mathrm{He}$ & $\mathrm{a} 0$ \\
$\mathrm{CO}$ & $\mathrm{H}_{2}, \mathrm{He}$ & $\mathrm{a} 0$ \\
$\mathrm{NO}$ & air & $\mathrm{a} 0$ \\
$\mathrm{H}_{2} \mathrm{O}$ & $\mathrm{H}_{2}, \mathrm{He}$, self, air & $\mathrm{a} 0, \mathrm{a} 1$ \\
$\mathrm{CO}_{2}$ & air, self & $\mathrm{a} 0$ \\
$\mathrm{SO}_{2}$ & $\mathrm{H}, \mathrm{He}$ & $\mathrm{a} 0, \mathrm{a} 5$ \\
$\mathrm{HCN}$ & $\mathrm{He}, \mathrm{He}$ & $\mathrm{a} 0$ \\
$\mathrm{OCS}$ & $\mathrm{He}, \mathrm{He}$ & $\mathrm{a} 0$ \\
$\mathrm{PH}_{3}$ & $\mathrm{He}, \mathrm{He}$ & $\mathrm{a} 0, \mathrm{c} 1$ \\
$\mathrm{NH}_{3}$ & $\mathrm{He}, \mathrm{He}$ & $\mathrm{a} 0$ \\
$\mathrm{H}_{2} \mathrm{CO}$ & $\mathrm{He}, \mathrm{He}$ & $\mathrm{a} 0$ \\
$\mathrm{C}_{2} \mathrm{H}_{2}$ & $\mathrm{He}, \mathrm{He}$ & $\mathrm{a} 0$ \\
$\mathrm{CH}_{4}$ & $\mathrm{He}, \mathrm{He}, \mathrm{CO}_{2}$ & $\mathrm{a} 0, \mathrm{a} 1$ \\
\hline
\end{tabular}

The . broad file has a hierarchical structure; each record starts with four compulsory columns: a label defining the broadening scheme ('a0', 'a1',...), values of $\gamma_{\text {ref }}, n$ and $J^{\prime \prime}$. The compulsory fields are followed by additional quantum numbers when a more detailed specification of the quantum assignments is available. The most basic scheme 'a0' represents broadening with $J^{\prime \prime}$ dependence only. The additional basic scheme 'a1' is used for the case with the $\left(J^{\prime}, J^{\prime \prime}\right)$ dependence. Any other schemes (e.g. 'a2', 'a3', 'a4', 'b1', 'b2', 'c1', 'c2') are molecule specific and should be described as part of the line list specifications. The broadening data format is illustrated in Table 9. 
Table 9

Example of the three basic broadening schemes in the ExoMol diet, 'a0' and 'a1'.

\begin{tabular}{lllll}
\hline Label & $\gamma$ & $n$ & $J^{\prime \prime}$ & $J^{\prime}$ \\
\hline $\mathrm{a} 0$ & 0.0860 & 0.096 & 0 & \\
$\mathrm{a} 0$ & 0.0850 & 0.093 & 1 & \\
$\ldots$ & & & & \\
$\mathrm{a} 1$ & 0.0860 & 0.096 & 0 & 1 \\
$\mathrm{a} 1$ & 0.0850 & 0.093 & 1 & 2 \\
$\ldots$ & & & & \\
\hline
\end{tabular}

The requirement for enhanced line broadening parameters was identified by Fortney et al. [261] in their study on the need for laboratory data requirements for studies of exoplanetary atmospheres. Recognising this, our plan is to significantly enhance the treatment of pressure effects in future releases of the ExoMol database.

\subsection{ExoMolOP: Opacities}

Recently Chubb et al. [262] computed opacity cross sections and $k$-tables for all molecules available from the ExoMol database plus some atomic data from NIST. These data are formatted for use in various retrieval codes including Tau-REx [13-15], ARCiS [24], petitRADTRANS [28], and NEMESIS [17]. Data were calculated on temperature-pressure grids suitable for characterising a variety of exoplanet and stellar atmospheres. Broadening parameters were taken from the literature where available, with broadening parameters used for a known molecule with a similar dipole moment, where they are not available. Tables of cross sections and $k$-coefficients are provided by ExoMol as part of the line list webpage. The format of the data and the resolution (grid spacing) is application dependent, for example the opacities for ARCiS, petitRADTRANS, and NEMESIS are given as $k$-tables at the resolving power of $R=1000$ while Tau-REx works with cross-sections with $R=15000$.

The opacity cross sections and $k$-tables will also be made available via the virtual atomic and molecular data centre (VAMDC) portal $[263,264]$. We note that ExoMol data have also been extensively used to construct the EXOPLINES molecular absorption crossSection database for brown dwarf and giant exoplanet atmospheres [265].

\subsection{Post-processing}

ExoMol provides post-processing capabilities through the program ExoCross [266]. ExoCross has many functions such as generating pressure and temperature dependent cross sections, partition functions, specific heat, state-resolved radiative lifetimes, non-LTE spectra, electric dipole, electric quadrupole and magnetic dipole spectra. ExoCross can read data in both ExoMol and HITRAN [267] formats and output it in these formats as well as SPECTRA (http://spectra.iao.ru/) and Phoenix formats [16]. Should data be needed in say HITRAN format, it is strongly recommended that the data are downloaded to a local computer in the much more compact ExoMol format and then processed using ExoCross. Examples of ExoCross input files are provided on the website.

There are also a number of Python utilities available on the ExoMol website. These are now largely redundant as ExoCross provides all functionality required to work with ExoMol line lists. However, we mention them for completeness: Utility extract_trans.py reads the .trans in .bz2 format without requiring it to be uncompressed. The ExoMol_to_HITRAN.py script converts ExoMol format to HITRAN format; this should be used with caution as ExoMol format is significantly more compact than HITRAN format and the line list files are large. The program exomol2gf.py can be used to generate oscillator strengths.

\subsection{New web services}

New web services include:

- A molecule and line list search.

- A graphical illustration of a line list represented by absorption spectra at two or three temperatures (e.g. $300 \mathrm{~K}, 2000 \mathrm{~K}$ and $5000 \mathrm{~K}$ ) computed using a Doppler line profile on a grid of $1 \mathrm{~cm}^{-1}$.

- The ExoMol bibliography in the BibTeX format is stored at and version controlled by GitHub, see https://github.com/ExoMol/ bib.

\section{ExoMol data formats and API}

\subsection{Format of the States and Trans files}

The formats of the States and Transitions files are specified in Table 10 and 11 . Tables 12 and 13 show typical examples of the States (.states) and Transitions (.trans) files. A significant new feature of the 2020 update is the uncertainty field $\left(\mathrm{cm}^{-1}\right)$ in the States file appearing as column 5 after the $J$ values; this is currently an optional feature but will become a compulsory column for the ExoMol States file. The uncertainty values typically come from three different sources: (i) the uncertainties of the MARVEL energies $\left(\sim 0.000001-0.1 \mathrm{~cm}^{-1}\right)$, (ii) uncertainties obtained as the fitting observed - calculated error of energies as part of the refinement to experimental data (energies or line positions) participated in the refinement $\left(\sim 0.001-1 \mathrm{~cm}^{-1}\right)$ and (iii) roughly estimated uncertainties for all other states that cannot be verified against existing experiment $\left(\sim 0.1-10 \mathrm{~cm}^{-1}\right)$. The uncertainty in the States file are given using the same format as the energy term values, i.e. with six decimal places after the decimal point, see Table 10.

To date the new standard of the States file has being applied to only a number of key/recent line lists namely $\mathrm{H}_{2} \mathrm{O}$ (POKAZATEL) [61], AlH (AlHambra) [122], C 2 (8states) [124,194], HCCH (aCeTY) [130], $\mathrm{CO}_{2}$ (UCL-4000) [132], $\mathrm{H}_{3} \mathrm{O}^{+}$(eXeL) [133] and TiO (Toto) [126]. A rolling programme is in place for updating the other files so the uncertainty is uniformly available for all sources.

Table 10

Updated specification of the ExoMol States file.

\begin{tabular}{llll}
\hline Field & Fortran Format & C Format & Description \\
\hline$i$ & I12 & $\% 12 \mathrm{~d}$ & State ID \\
$E$ & F12.6 & $\% 12.6 \mathrm{f}$ & State energy in $\mathrm{cm}^{-1}$ \\
$g_{\text {tot }}$ & $\mathrm{I} 6$ & $\% 6 \mathrm{~d}$ & State degeneracy \\
$J$ & $\mathrm{I} 7 / \mathrm{F} 7.1$ & $\% 7 \mathrm{~d} / \% 7.1 \mathrm{f}$ & $J$-quantum number (integer/half-integer) \\
$(\Delta E)$ & F12.6 & $\% 12.6 \mathrm{f}$ & Energy uncertainty in $\mathrm{cm}^{-1}$ (currently optional) \\
$(\tau)$ & ES12.4 & $\% 12.4 \mathrm{e}$ & Lifetime in s (optional) \\
$(g)$ & F10.6 & $\% 10.6 \mathrm{f}$ & Landé g-factor (optional) \\
$($ Extra $)$ & - & - & Extra quantum numbers, any format (optional) \\
\hline
\end{tabular}


Table 11

Specification of the Transitions file.

\begin{tabular}{llll}
\hline Field & Fortran Format & C Format & Description \\
\hline$i$ & $\mathrm{I} 12$ & $\% 12 \mathrm{~d}$ & Upper state ID \\
$f$ & $\mathrm{I} 12$ & $\% 12 \mathrm{~d}$ & Lower state ID \\
$A$ & ES10.4 & $\% 10.4 \mathrm{e}$ & Einstein $A$ coefficient in $\mathrm{s}^{-1}$ \\
$\left.\widetilde{(v}_{f i}\right)$ & E15.6 & $\% 15.6 \mathrm{e}$ & Transition wavenumber in $\mathrm{cm}^{-1}$ (optional). \\
\hline
\end{tabular}

Table 12

An example of an extract from the final States file for UCL-4000 of $\mathrm{CO}_{2}$ [132].

\begin{tabular}{lllllllllll}
\hline$i$ & $\tilde{E}$ & $g_{\text {tot }}$ & $J$ & unc. & $\Gamma$ & elf & $n_{1}$ & $n_{2}^{\text {lin }}$ & $l_{2}$ & $n_{3}$ \\
\hline 1 & 0.000000 & 1 & 0 & 0.000500 & $\mathrm{~A} 1$ & $\mathrm{e}$ & 0 & 0 & 0 & 0 \\
2 & 1285.408200 & 1 & 0 & 0.000500 & $\mathrm{~A} 1$ & $\mathrm{e}$ & 0 & 2 & 0 & 0 \\
3 & 1388.184200 & 1 & 0 & 0.005000 & $\mathrm{~A} 1$ & $\mathrm{e}$ & 1 & 0 & 0 & 0 \\
4 & 2548.366700 & 1 & 0 & 0.000500 & $\mathrm{~A} 1$ & $\mathrm{e}$ & 1 & 2 & 0 & 0 \\
5 & 2671.142957 & 1 & 0 & 0.005000 & $\mathrm{~A} 1$ & $\mathrm{e}$ & 2 & 0 & 0 & 0 \\
6 & 2797.136000 & 1 & 0 & 0.005000 & $\mathrm{~A} 1$ & $\mathrm{e}$ & 1 & 2 & 0 & 0 \\
7 & 3792.681898 & 1 & 0 & 0.005000 & $\mathrm{~A} 1$ & $\mathrm{e}$ & 1 & 4 & 0 & 0 \\
8 & 3942.541358 & 1 & 0 & 0.005000 & $\mathrm{~A} 1$ & $\mathrm{e}$ & 3 & 0 & 0 & 0 \\
9 & 4064.274256 & 1 & 0 & 0.005000 & $\mathrm{~A} 1$ & $\mathrm{e}$ & 3 & 0 & 0 & 0 \\
10 & 4225.096148 & 1 & 0 & 0.005000 & $\mathrm{~A} 1$ & $\mathrm{e}$ & 1 & 4 & 0 & 0 \\
11 & 4673.325200 & 1 & 0 & 0.000500 & $\mathrm{~A} 1$ & $\mathrm{e}$ & 0 & 0 & 0 & 2 \\
12 & 5022.349428 & 1 & 0 & 0.005000 & $\mathrm{~A} 1$ & $\mathrm{e}$ & 1 & 6 & 0 & 0 \\
13 & 5197.252900 & 1 & 0 & 0.005000 & $\mathrm{~A} 1$ & $\mathrm{e}$ & 3 & 2 & 0 & 0 \\
14 & 5329.645446 & 1 & 0 & 0.005000 & $\mathrm{~A} 1$ & $\mathrm{e}$ & 4 & 0 & 0 & 0 \\
15 & 5475.553054 & 1 & 0 & 0.000500 & $\mathrm{~A} 1$ & $\mathrm{e}$ & 3 & 2 & 0 & 0 \\
16 & 5667.644584 & 1 & 0 & 0.005000 & $\mathrm{~A} 1$ & $\mathrm{e}$ & 2 & 4 & 0 & 0 \\
17 & 5915.212302 & 1 & 0 & 0.000500 & $\mathrm{~A} 1$ & $\mathrm{e}$ & 0 & 2 & 0 & 2
\end{tabular}

$i$ : State counting number. $\tilde{E}$ : State energy in $\mathrm{cm}^{-1} \cdot g_{\text {tot }}$ : Total state degeneracy. J: Total angular momentum. unc.: Uncertainty in $\mathrm{cm}^{-1}$. $\Gamma$ : Total symmetry index in $\mathcal{C}_{2 \mathrm{v}}(\mathrm{M})$. e/f: Kronig rotationless parity. $n_{1}$ : Normal mode stretching symmetry $\left(A_{1}\right)$ quantum number. $n_{2}^{\text {lin }}$ : Normal mode linear molecule bending $\left(A_{1}\right)$ quantum number. $l_{2}$ : Normal mode vibrational angular momentum quantum number. $n_{3}$ : Normal mode stretching asymmetric $\left(B_{1}\right)$ quantum number.

Table 13

Extract from the Transitions file for $\mathrm{CaO}$.

\begin{tabular}{llll}
\hline$f$ & $i$ & $A_{f i}$ & $\tilde{v}_{f i}$ \\
\hline 10571 & 10884 & $9.5518 \mathrm{E}-06$ & 120.241863 \\
21053 & 21375 & $1.9515 \mathrm{E}-05$ & 120.242886 \\
8726 & 9672 & $1.8658 \mathrm{E}-04$ & 120.243522 \\
11655 & 11950 & $5.0065 \mathrm{E}-06$ & 120.243733 \\
93209 & 93967 & $5.7055 \mathrm{E}-03$ & 120.244192 \\
2228 & 3175 & $7.3226 \mathrm{E}-07$ & 120.244564 \\
46727 & 46432 & $1.0599 \mathrm{E}-04$ & 120.244658 \\
44436 & 44774 & $1.4626 \mathrm{E}-04$ & 120.245583 \\
29037 & 28723 & $1.8052 \mathrm{E}-04$ & 120.245669 \\
4458 & 4805 & $1.0431 \mathrm{E}-08$ & 120.246396 \\
69313 & 68434 & $5.0531 \mathrm{E}-06$ & 120.248178 \\
22640 & 22985 & $1.1281 \mathrm{E}-07$ & 120.248891 \\
57027 & 56721 & $7.1064 \mathrm{E}-06$ & 120.250180 \\
\hline
\end{tabular}

$f$ : Upper state counting number; $i$ : Lower state counting number; $A_{f}$ : Einstein-A coefficient in $\mathrm{s}^{-1}$; $\tilde{v}_{f i}:$ transition wavenumber in $\mathrm{cm}^{-1}$.

\subsection{Formats for other data types}

Table 14 shows the format of the . broad files containing the line broadening parameters. Table 15 shows the format of the cross section . cross and super-lines . super files. Tables 16 and 17 show the format of the partition function .pf and specific heat . cp files.

\subsection{API}

\subsubsection{Searching for data through the API}

The molecules and isotopologues available in ExoMol are listed in a master file located at: www.exomol.com/exomol.all. Given a molecule or list of isotopologues, ExoMol can be searched for rec-

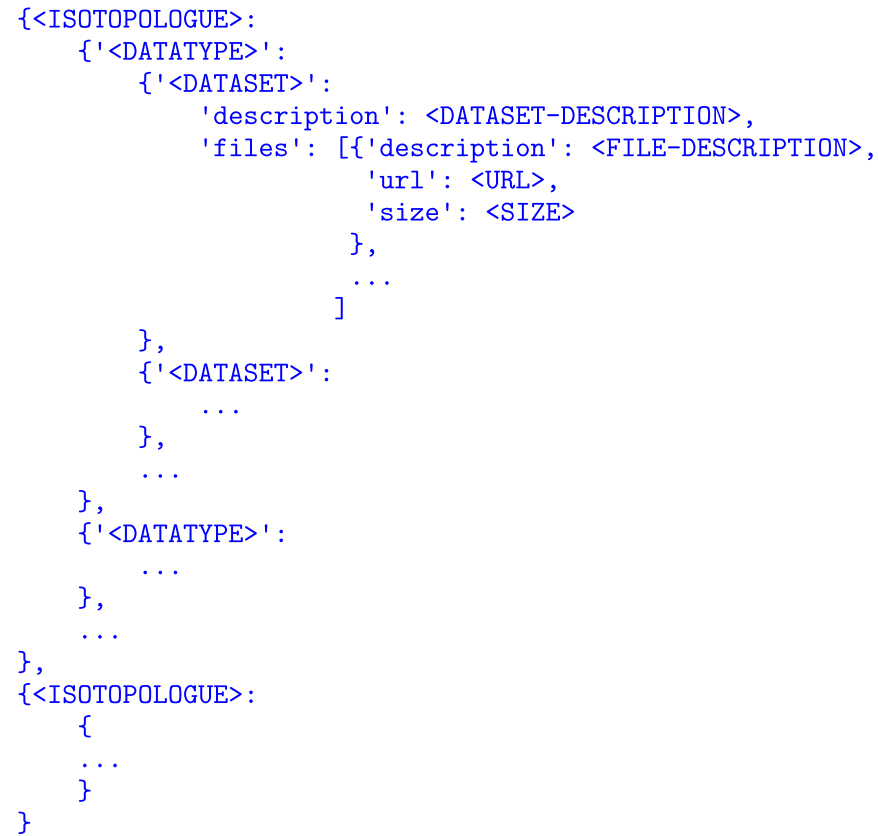

Fig. 4. A JSON API structure used for search queries using the HTTP GET request in ExoMol.

ommended datasets using the API which can be queried using the HTTP GET request method described below. The structure of a JSON file is illustrated in Fig. 4.

To search for all data sets (and their files) related to a single molecule, use the query:

http://exomol.com/api?molecule=〈MOLECULE $\rangle$ 
Table 14

Specification of the mandatory part of the pressure broadening parameters file.

\begin{tabular}{llll}
\hline Field & Fortran Format & C Format & Description \\
\hline code & A2 & $\% 2 \mathrm{~s}$ & Code identifying quantum number set following $J^{\prime \prime}$ \\
$\gamma_{\text {ref }}$ & F6.4 & $\% 6.4 \mathrm{f}$ & Lorentzian half-width at reference temperature and pressure in $\mathrm{cm}^{-1} / \mathrm{bar}$ \\
$n$ & F6.3 & $\% 6.3 \mathrm{f}$ & Temperature exponent \\
$J^{\prime \prime}$ & I7/F7.1 & $\% 7 \mathrm{~d} / \% 7.1 \mathrm{f}$ & Lower $J$-quantum number integer/half-integer \\
\hline
\end{tabular}

Fortran format, $J$ integer: (A2, 1x, F6.4,1x,F6.3,1x, I7) or $J$ half-integer: $(\mathrm{A} 2,1 \mathrm{x}, \mathrm{F} 6.4,1 \mathrm{x}, \mathrm{F} 6.3,1 \mathrm{x}, \mathrm{F} 7.1)$

Table 15

Specification of the . cross cross section and .stick file format.

\begin{tabular}{llll}
\hline Field & Fortran Format & C Format & Description \\
\hline$\tilde{v}_{i}$ & F12.6 & $\% 12.6 \mathrm{f}$ & Central bin wavenumber, $\mathrm{cm}^{-1}$ \\
$\sigma_{i}$ & ES14.8 & $\% 14.8 \mathrm{e}$ & Absorption cross section, $\mathrm{cm}^{2} \mathrm{molec}^{-1}$ \\
$\alpha_{i}$ & ES14.8 & $\% 14.8 \mathrm{e}$ & Absorption coefficient, cm molec \\
\hline
\end{tabular}

Fortran format: (F12.6,1x, ES14.8)

For example, the URL http://exomol.com/api?molecule=NH3 returns a JSON data structure describing all files related to the ammonia molecule. Within this, the list of files belonging to the BYTe15 line list for ${ }^{15} \mathrm{~N}^{1} \mathrm{H}_{3}$ is accessible by traversing the JSON object with:

json_result ['(15N)(1H)3'] ['linelist ']

['BYTe-15']['files'] and the URL for the States file belonging to this line list is at:

jsonresult ['(15N)(1H) 3'] ['linelist']

['BYTe-15'] ['files'] [1] ['url'].

Note that molecules are identified by simple text strings (no subscript or superscript symbols) but that special characters must be URL-encoded: for example, the $\mathrm{HeH}^{+}$cation is identified by molecule $=\mathrm{HeH}_{\%} 2 \mathrm{~B}$. Most software will provide this "percentencoding" automatically.

Searches can be further refined by isotopologue by setting the query keyword isotopologues to a comma-delimited sequence; for example:

http://exomol.com/api/?isotopologues=(12C)(32S),(12C)(34S)

Restricting the search by datatype is also supported:

http://exomol.com/api/?molecule=H2O\&datatype=linelist returns the details of linelists for the isotopologues of water, omitting partition functions, cooling functions, opacities, etc. Valid values for the datatype parameter are: linelist, energylevels, opacity, super ("Super" line lists), Cp (heat capacity), broadening_coefficients, coolingfunction, partitionfunction.

\subsubsection{Accessing the data via the API}

There are a number of ways of accessing the data. First, all data sets are available on the ExoMol website (www.exomol.com) and can be downloaded manually. Many ExoMol line lists contain in excess of 10 billion transitions. In this case the file of transitions are generally split into chunks in frequency. These files are compressed using . bz2 format.

However, if multiple datasets are required manual downloads are inefficient. The systematic structure of the ExoMol database filesystem allows for automated downloads from within software
Table 17

Specification of the . $\mathrm{cp}$ specific heat file.

\begin{tabular}{llll}
\hline Field & Fortran Format & C Format & Description \\
\hline$T$ & F8.1 & $\% 8.1 \mathrm{~d}$ & Temperature in $\mathrm{K}$ \\
$C_{p}(T)$ & F15.4 & $\% 15.4 \mathrm{~d}$ & Specific heat function (dimensionless). \\
\hline
\end{tabular}

Fortran format: (F8.1,1x,F15.4)

or using utilities such as wget and curl. As described in detail in the ExoMol2016 release, the API structures the ExoMol data resource files as URLs of the form:

http://exomol.com/db/〈MOLECULE $\rangle /\langle$ ISOTOPOLOGUE $\rangle$

$\mid\langle$ DATASET $\rangle /\langle$ FILENAME $\rangle$ where $<$ FILENAME $>$ is structured ' $<$ ISOTOPOLOGUE $>$ _- $<$ DATASET $>$. $<$ DATATYPE $>$ '. For example, the States file of the YYLT line list for ${ }^{31} \mathrm{P}^{15} \mathrm{~N}$ is obtainable at the URL http://exomol.com/db/PN/31P-15N/YYLT/ 31P-15N__YYLT.states.bz2.

Furthermore, each dataset has a manifest that lists the data files it is comprised of and their sizes (in bytes). The manifest is a single text file located at the above URL with the filename ' $<$ ISOTOPOLOGUE $>$ __ $<$ DATASET $>$. manifest '. For example, the contents of http://exomol.com/db/PN/31P-15N/YYLT/31P-15N_ YYLT.manifest are:

http://exomol.com/db/PN/31P-15N/YYLT/31P-15N_YYLT.states. bz2 142161 http://exomol.com/db/PN/31P-15N/YYLT/31P-15N_YYLT. trans.bz2 1803083 http://exomol.com/db/PN/31P-15N/YYLT/ 31P-15N_YYLT.pf 175000 http://exomol.com/db/PN/31P-15N/ YYLT/31P-15N_YYLT.def 4761 http://exomol.com/db/PN/31P-15N/ YYLT/31P-15N_YYLT.manifest 323

\section{Moving to higher spectral resolution}

Transit spectroscopy of exoplanets has thus far been performed at rather low resolution; however, very precise spectroscopic data are required for high resolution Doppler spectroscopy [277-279]. This has proved to be an issue for important species [70]. Indeed the ExoMol datasets described above were generally constructed with greater emphasis on completeness than obtaining very precise transition frequencies [280]. In practice, however, the use of empirical energy levels in the States file means that some transition frequencies are indeed reproduced with high (experimental) accuracy; however, with the ExoMol2016 data structure it was not possible to tell how accurately a particular transition was predicted.

Although some work has been done on using ExoMol data to provide the input for high resolution studies [214], there is a clear need to adapt the database to provide the laboratory data needed

Table 16

Specification of the $\cdot \mathrm{pf}$ partition function file.

\begin{tabular}{llll}
\hline Field & Fortran Format & C Format & Description \\
\hline$T$ & F8.1 & $\% 8.1 \mathrm{~d}$ & Temperature in K \\
$Q(T)$ & F15.4 & $\% 15.4 \mathrm{~d}$ & Partition function (dimensionless). \\
$W(T)$ & ES12.4 & $\% 12.4 \mathrm{e}$ & Cooling function in ergs s molecule $^{-1}$ (if available). \\
\hline
\end{tabular}

Fortran format: (F8.1,1x,F15.4,1x,ES12.4) or (F8.1,1x, F15.4) 
Table 18

Molecules of importance for the ExoMol project with published MARVEL datasets.

\begin{tabular}{lllll}
\hline Molecule & $N_{\text {iso }}$ & $N_{\text {elec }}$ & $N_{\text {levels }}$ & Reference(s) \\
\hline $\mathrm{H}_{2} \mathrm{O}$ & 7 & 1 & 18486 & Tennyson et al. [217,223,224,268] \\
update & 1 & 1 & 19200 & Tóbiás et al. [269], Furtenbacher et al. [270] \\
$\mathrm{H}_{3}^{+}$ & 3 & 1 & 652 & Furtenbacher et al. [231,271] \\
$\mathrm{NH}_{3}$ & 1 & 1 & 4951 & Al Derzi et al. [188] \\
update & 1 & 1 & 4936 & Furtenbacher et al. [272] \\
$\mathrm{C}_{2}$ & 1 & 14 & 5699 & Furtenbacher et al. [193] \\
update & 1 & 20 & 7087 & McKemmish et al. [194] \\
$\mathrm{TiO}$ & 1 & 9 & 10564 & McKemmish et al. [126,199] \\
$\mathrm{HCCH}$ & 1 & 1 & 11213 & Chubb et al. [235] \\
$\mathrm{SO} \mathrm{H}_{2}$ & 3 & 1 & 15130 & Tóbiás et al. [234] \\
$\mathrm{H}_{2} \mathrm{~S}$ & 1 & 1 & 11213 & Chubb et al. [230] \\
$\mathrm{ZrO}$ & 1 & 10 & 8088 & McKemmish et al. [273] \\
$\mathrm{O}_{2}$ & 1 & 6 & 4279 & Furtenbacher et al. [274] \\
$\mathrm{NH}$ & 1 & 4 & 1058 & Darby-Lewis et al. [282] \\
$\mathrm{CaOH}$ & 1 & 5 & 1954 & Wang et al. [275] \\
$\mathrm{H}_{2} \mathrm{CO}$ & 1 & 1 & 4841 & Al-Derzi et al. [236] \\
$\mathrm{NO}$ & 1 & 1 & 4106 & Wong et al. [115] \\
$\mathrm{AlH}$ & 2 & 2 & 331 & Yurchenko et al. [122] \\
$\mathrm{BeH}$ & 3 & 2 & 1264 & Darby-Lewis et al. [172] \\
$\mathrm{CN}$ & 1 & 10 & 7779 & Syme and McKemmish [276]
\end{tabular}

$N_{\text {iso }}$ Number of isotopologues considered; $N_{\text {elec }}$ Number of electronic states considered;

$N_{\text {levels }}$ Number of energy levels extracted: value is for the main isotopologue.

for these studies. For this reason we have updated the data structure to allow the uncertainty in a particular transition frequency to be determined. This is done via uncertainties in the energy levels which are now specified (optionally) in the States file, see Table 10.

To improve the accuracy of the predicted spectra, the ExoMol States files are being systematically updated using empiricallydetermined energy levels. The main means of doing this is via the MARVEL (measured active rotational-vibrational energy levels) procedure [190,269,281]. MARVEL inverts available high resolution spectra for a given isotopologue to give a list of empirical energy levels with associated uncertainties. For levels determined by MARVEL these uncertainties are now given in the States file. Otherwise the (usually much larger) uncertainty arising from the calculation used to generate the line list is given. By combining the uncertainty of upper and lower states $\left(\Delta E_{u}, \Delta E_{l}\right)$ using the standard formula

$\Delta E_{v}=\frac{1}{\sqrt{2}} \sqrt{\left(\left(\Delta E_{u}\right)^{2}+\left(\Delta E_{l}\right)^{2}\right)}$

gives the uncertainty in the transition wavenumber, $\Delta E_{v}$.

To help improve the accuracy of key line lists we have been running MARVEL projects on relevant molecules. Table 18 lists the astronomically important molecules for which MARVEL studies have been completed. We note that a number of these studies $[199,230,235,273,282]$ have been performed as part of the so-called ORBYTS schools outreach project, see Sousa-Silva et al. [283] for a discussion of this. We are in the process of working through all the molecules in the ExoMol database running MARVEL projects for those isotopologues for which there are enough high accuracy laboratory data available to justify this activity.

\section{Other future development}

The ExoMol project already maintains extensive molecule by molecule bibliography files. These are stored on LaTeX's BibTex format and are freely accessible at https://github.com/ExoMol/bib/ tree/master/exomol. Our aim is to make referencing to the original data as easy and convenient as possible, including automatic generation of the list of references to cite in the appropriate format such as BibTex, Endnote etc. Such services have been developed and offered by HITRAN [284].
We are in the process of moving the database to a more powerful platform. After the move we plan to offer the ability to compute cross sections for a given species, temperature and pressure on the fly. This will be done using super-lines which greatly reduce the computing time. We will also facilitate the computation of $k$-tables for a given atmospheric model.

Finally the move should allow much greater integration with the code Tau-REx $[13,14]$. Tau-REx is an open source retrieval code for exoplanetary atmospheres which has just undergone a major upgrade [15]. Integration with the Tau-REx-III upgrade via a graphical interface is currently in progress.

\section{Conclusions}

The ExoMol database presented here is a molecule-by-molecule set of comprehensive line lists for modelling spectra and other properties of hot gases. The choice of molecules is dictated by the need to model the atmospheres of exoplanets and other hot astronomical objects, but the spectroscopic data have much wider applications than this. We are still in the process of adding molecules to the database and are receptive to suggestions of other key species to include. In addition we are working on improving the accuracy of the line positions, particularly for strong lines, enhancing the treatment of pressure broadening, and extending the range of wavelengths covered into the ultra-violet for molecules where this is considered important. In addition, we also plan to expand the database to consider temperature dependent photodissociation.

\section{Declaration of Competing Interest}

The authors declare that they have no known competing financial interests or personal relationships that could have appeared to influence the work reported in this paper.

\section{Acknowledgments}

We thank the many scientists who have contributed directly or indirectly to the ExoMol project including Ala'a Azzam, Emma Barton, Bob Barber, Phillip Coles, Lorenzo Lodi, Barry Mant, Andrei Patrascu, Anatoly Pavlyuchko, Clara Sousa-Silva, Tom Rivlin, Daniel Underwood, Andrei Yachmenev and Peter Bernath. This work was 
supported by the European Research Council (ERC) under the Advanced Investigator ExoMol Project 267219 and the ExoAI project 758892, STFC through grants ST/H008586/1 and ST/K00333X/1 and the European Union's Horizon 2020 Research and Innovation Programme, under Grant Agreement 776403.

\section{Supplementary material}

Supplementary material associated with this article can be found, in the online version, at doi:10.1016/j.jqsrt.2020.107228.

\section{References}

[1] Tennyson J, Yurchenko SN. ExoMol: molecular line lists for exoplanet and other atmospheres. Mon Not R Astron Soc 2012;425:21-33. doi:10.1111/j. 1365-2966.2012.21440.x

[2] Tinetti G, Encrenaz T, Coustenis A. Spectroscopy of planetary atmospheres in our galaxy. Astron Astrophys Rev 2013;21:1-65. doi:10.1007/ s00159-013-0063-6.

[3] Bernath PF. Molecular astronomy of cool stars and sub-stellar objects. Int Rev Phys Chem 2009;28:681-709. doi:10.1080/01442350903292442.

[4] Allard F, Hauschildt PH, Alexander DR, Starrfield S. Model atmosphere of very low mass stars and brown dwarfs. Ann Rev Astron Astrophys 1997;35:137-77.

[5] Bordbar MH, Wecel G, Hyppanen T. A line by line based weighted sum of gray gases model for inhomogeneous $\mathrm{CO}_{2}-\mathrm{H}_{2} \mathrm{O}$ mixture in oxy-fired combustion. Cumbust Flame 2014;161:2435-45. doi:10.1016/j.combustflame.2014.03. 013

[6] Consalvi JL, Liu F. A database of narrow-band parameters for fuels commonly encountered in fire applications. Fire Saf J 2015;78:202-18. doi:10. 1016/j.firesaf.2015.10.002

[7] Chu YN, Wang HM, Li JQ, Cheng P, Cao DZ. Emission observation for electronically excited state $\mathrm{SO}\left(\mathrm{c}^{1} \Sigma^{-}\right)$in gas phase. Chem Phys Lett 2002;366:147-52. doi:10.1016/S0009-2614(02)01525-7.

[8] Carney JR, Lightstone JM, Piecuch S, Koch JD. Water temperature and concentration measurements within the expanding blast wave of a high explosive. Measure Sci Tech 2011;22:045601. doi:10.1088/0957-0233/22/4/045601.

[9] Evseev V, Fateev A, Clausen S. High-resolution transmission measurements of $\mathrm{CO}_{2}$ at high temperatures for industrial applications. J Quant Spectrosc Radiat Transf 2012;113:2222-33. doi:10.1016/j.jqsrt.2012.07.015.

[10] Dello Russo N, DiSanti MA, Magee-Sauer K, Gibb EL, Mumma MJ, Barber RJ, et al. Accurate rotational temperature retrievals from hot-band line near 2.9 $\mu \mathrm{m}$. Icarus 2004;168:186-200.

[11] Dello Russo N, Bonev BP, DiSanti MA, Gibb EL, Mumma MJ, Magee-Sauer K, et al. Water production rates, rotational temperatures and spin temperatures in comets C/1999 H1 (Lee), C/1999 S4 and C/2001 A2. Astrophys J 2005;621:537-44.

[12] Gray MD, Baudry A, Richards AMS, Humphreys EML, Sobolev AM, Yates JA. The physics of water masers observable with ALMA and SOFIA: model predictions for evolved stars. Mon Not R Astron Soc 2016;456:374-404. doi:10. 1093/mnras/stv2437

[13] Waldmann IP, Tinetti G, Barton EJ, Yurchenko SN, Tennyson J. Tau-REx I: a next generation retrieval code for exoplanetary atmospheres. Astrophys J 2015;802:107. doi:10.1088/0004-637X/802/2/107.

[14] Waldmann IP, Rocchetto M, Tinetti G, Barton EJ, Yurchenko SN, Tennyson J. $\tau$-REx II: retrieval of emission spectra. Astrophys I 2015:813:13.

[15] Al-Refaie AF, Changeat Q, Waldmann IP, Tinetti G. TauREx III: a fast, dynamic and extendable framework for retrievals 2019. arXiv: 1912.07759.

[16] Jack D, Hauschildt PH, Baron E. Time-dependent radiative transfer with PHOENIX. Astron Astrophys 2009;502:1043-9.

[17] Irwin PGJ, Teanby NA, de Kok R, Fletcher LN, Howett CJA, Tsang CCC, et al. The NEMESIS planetary atmosphere radiative transfer and retrieval tool. J Quant Spectrosc Radiat Transf 2008;109:1136-50. doi:10.1016/j.jqsrt.2007.11.006.

[18] Line MR, Wolf AS, Zhang X, Knutson H, Kammer JA, Ellison E, et al. A systematic retrieval analysis of secondary eclipse spectra. i. a comparison of atmospheric retrieval techniques. Astrophys J 2013;775:137.

[19] Malik M, Grosheintz L, Mendonça JM, Grimm SL, Lavie B, Kitzmann D, et al. HELIOS: An open-source, GPU-accelerated radiative transfer code for selfconsistent exoplanetary atmospheres. Astrophys J 2017;153:56. doi:10.3847| $1538-3881 / 153 / 2 / 56$.

[20] Kitzmann D, Heng K, Oreshenko M, Grimm SL, Apai D, Bowler BP, et al. Helios-r2: a new bayesian, open-source retrieval model for brown dwarfs and exoplanet atmospheres. Astrophys J 2020;890:174. doi:10.3847/1538-4357/ ab6d71.

[21] Drummond B, Tremblin P, Baraffe I, Amundsen DS, Mayne NJ, Venot O, et al. The effects of consistent chemical kinetics calculations on the pressuretemperature profiles and emission spectra of hot Jupiters. Astron Astrophys 2016;594:A69. doi:10.1051/0004-6361/20162.8799.

[22] Goyal JM, Mayne N, Sing DK, Drummond B, Tremblin P, Amundsen DS, et al. A library of ATMO forward model transmission spectra for hot Jupiter exoplanets. Mon Not R Astron Soc 2018;473:5158-85. doi:10.1093/mnras/stx3015.
[23] Phillips MW, Tremblin P, Baraffe I, Chabrier G, Allard NF, Spiegelman F, et al. A new set of atmosphere and evolution models for cool T-Y brown dwarfs and giant exoplanets. Astron Astrophys 2020;637:A38. doi:10.1051/ 0004-6361/201937381.

[24] Min M, Ormel CW, Chubb KL, Helling C, Kawashima Y. The ARCis framework for exoplanet atmospheres: modelling philosophy and retrieval. Astron Astrophys 2020. (In preparation)

[25] Stolker T, Min M, Stam DM, Molliere P, Dominik C, Waters LBFM. Polarized scattered light from self-luminous exoplanets three-dimensional scattering radiative transfer with ARTES. Astron Astrophys 2017;607:A42. doi:10.1051/ 0004-6361/201730780.

[26] Gandhi S, Madhusudhan N. Retrieval of exoplanet emission spectra with HyDRA. Mon Not R Astron Soc 2018;474:271-88. doi:10.1093/mnras/stx2748.

[27] Gandhi S, Madhusudhan N. GENESIS: New self-consistent models of exoplanetary spectra. Mon Not R Astron Soc 2017;472:2334-55. doi:10.1093/mnras/ stx1601.

[28] Molliére P, Wardenier JP, van Boekel R, Henning T, Molaverdikhani K, Snellen IAG. petitRADTRANS - A python radiative transfer package for exoplanet characterization and retrieval. Astron Astrophys 2019;627:A67. doi:10. 1051/0004-6361/201935470.

[29] Zhang M, Chachan Y, Kempton EMR, Knutson HA. Forward modeling and retrievals with PLATON, a fast open-source tool. Publ Astron Soc Pac 2019;131(997):034501. doi:10.1088/1538-3873/aaf5ad.

[30] Bailey J, Kedziora-Chudczer L. Modelling the spectra of planets, brown dwarfs and stars using VSTAR. Mon Not R Astron Soc 2012;419:1913-29. doi:10.1111/ j.1365-2966.2011.19845.x.

[31] Yurchenko SN, Tennyson J, Bailey J, Hollis MDJ, Tinetti G. Spectrum of hot methane in astronomical objects using a comprehensive computed line list. Proc Nat Acad Sci 2014;111:9379-83. doi:10.1073/pnas.1324219111.

[32] BART: Bayesian atmospheric radiative transfer code. Astrophys J Suppl 2020.

[33] Cubillos P.E., Yet another open-source radiative-transifer code for exoplanet modeling. 2020.

[34] Irwin PGJ, Bowles N, Braude AS, Garland R, Calcutt S, Coles PA, et al. Analysis of gaseous ammonia $\left(\mathrm{NH}_{3}\right)$ absorption in the visible spectrum of Jupiter update. Icarus 2019;321:572-82. doi:10.1016/j.icarus.2018.12.008.

[35] Irwin PGJ, Toledo D, Garland R, Teanby NA, Fletcher LN, Orton GS, et al. Probable detection of hydrogen sulphide $\left(\mathrm{H}_{2} \mathrm{~S}\right)$ in Neptune's atmosphere. Icarus 2019;321:550-63. doi:10.1016/j.icarus.2018.12.014.

[36] Berezhnoy AA, Borovicka J, Santos J, Rivas-Silva JF, Sandoval L, Stolyarov AV, et al. The $\mathrm{CaO}$ orange system in meteor spectra. Planet Space Sci 2018;151:27-32. doi:10.1016/j.pss.2017.10.007.

[37] Kraus M, Oksala ME, Cidale LS, Arias ML, Torres AF, Borges Fernandes M. Discovery of $\mathrm{SiO}$ band emission from galactic $<$ upper-case $>[$ Be supergiants. Astrophys J Lett 2015;800:L20. doi:10.1088/2041-8205/800/2/L20.

[38] Pavlenko YV. SiO bands in the infrared spectra of late-type stars. Kinemat Phys Celest Bodies 2019.

[39] Evans A, Pavlenko YV, Banerjee DPK, Munari U, Gehrz RD, Woodward CE, et al. Gas phase $\mathrm{SiO}$ in the circumstellar environment of the recurrent nova T Coronae Borealis. Mon Not R Astron Soc 2019;486:3498-505. doi:10.1093/ mnras/stz1071.

[40] Hughes GB, Lubin P, Meinhold P, O’Neill H, Brashears T, Zhang Q, et al. Standoff molecular composition analysis. In: Taylor EW, Cardimona DA, editors. Nanophotonics and macrophotonics for space environments IX; vol. 9616 of Proceedings of SPIE; 2015. p. 961603. doi:10.1117/12.2186795.

[41] Hughes GB, Lubin P, Cohen A, Madajian J, Kulkarni N, Zhang Q, et al. Remote laser evaporative molecular absorption spectroscopy. In: Hughes GB, editor. Planetary defense and space environment applications; 2016. Vol. 9981 of Proc. SPIE. SPIE, doi: $10.1117 / 12.2242730$.

[42] Pavlenko YV, Yurchenko SN, McKemmish LK, Tennyson J. Analysis of the TiO isotopologues in stellar optical spectra. Astron Astrophys 2020.

[43] Owens A, Yurchenko SN, Špirko V. Anomalous phosphine sensitivity coefficients as probes for a possible variation of the proton-to-electron mass ratio. Mon Not R Astron Soc 2018;473:4986-92. doi:10.1093/mnras/stx2696.

[44] Syme AM, Mousley A, Cunningham M, McKemmish LK. Diatomic rovibronic transitions as potential probes for proton-to-electron mass ratio across cosmological time. Australian J of Chem 2020. doi:10.1071/CH19448.

[45] Owens A, Zak EJ, Chubb KL, Yurchenko SN, Tennyson J, Yachmenev A. Simulating electric-field interactions with polar molecules using spectroscopic databases. Sci Rep 2017;45068:7. doi:10.1038/srep45068.

[46] Owens A, Yachmenev A. Richmol: a general variational approach for rovibrational molecular dynamics in external electric fields. J Chem Phys 2018;148(12):124102.

[47] Buchanan Z, Martin-Drumel MA, Eliet S, Turut J, Mouret G, Hindle F, et al. Building a database for QCL pumped far-IR lasers 74th international symposium on molecular spectroscopy; 2019. Doi:10.15278/isms.2019.MJ10

[48] Essig R, Pérez-Ríos J, Ramani H, Slone O. Direct detection of nuclear scattering of sub-GeV dark matter using molecular excitations. Phys Rev Res 2019;1:033105. doi:10.1103/PhysRevResearch.1.033105.

[49] Markov N. A quantum propulsion method. In: Georgiev P, Soares CG, editors. Sustainable development and innovations in marine technologies; vol. 3 of Proceedings in marine technology and ocean engineering. Maritime Assoc Mediterranean; Tech Univ Varna; 2020. p. 247-51. 18th International Congress of the Maritime-Association-of-the-Mediterreanean (IMAM), Varna, BULGARIA, SEP 09-11, 2019 ISBN 978-0-367-81008-5; 978-0-367-40951-7

[50] Joshi GC, Punetha LM, Pande MC. (A-X) system of SiO in sunspots. Solar Phys 1979;62:77-82. doi:10.1007/BF00150133. 
[51] Sriramachandran P, Ambika C, Shanmugavel R. The rotational temperatures of silicon hydride isotopomer lines in sunspot umbrae. New Astron 2012;17, :281-4. doi:10.1016/j.newast.2011.07.008.

[52] Joshi GC, Pande MC. On the presence of SH in the sunspot spectrum. Sol Phys 1979;62:69-75. doi:10.1007/BF00150132.

[53] Shanmugavel R, Bagare SP, Rajamanickam N, Balachandra Kumar K. Identification of beryllium hydride isotopomer lines in sunspot umbral spectra. Serb Astron J 2008;176:51-8. doi:10.2298/SAJ0876051S.

[54] Sriramachandran P, Bagare SP, Rajamanickam N, Balachandrakumar K. Presence ofLaO, ScO and VO molecular lines in sunspot umbral spectra. Sol Phys 2008;252:267-81. doi:10.1007/s11207-008-9261-1.

[55] Sotirovski P. The molecular spectrum of sunspot umbrae. Astron Astrophys 1971;14:319.

[56] Polyansky OL, Zobov NF, Viti S, Tennyson J, Bernath PF, Wallace L. Water in the sun: line assignments based on variational calculations. Science 1997;277:346-9.

[57] Polyansky OL, Zobov NF, Viti S, Tennyson J, Bernath PF, Wallace L. High temperature rotational transitions of water in sunspot and laboratory spectra. J Mol Spectrosc 1997; 186:422-47.

[58] Zobov NF, Polyansky OL, Tennyson J, Shirin SV, Nassar R, Hirao T, et al. Using laboratory spectroscopy to identify lines in the $\mathrm{K}$ and L-band spectrum of water in a sunspot. Astrophys J 2000;530:994-8.

[59] Tereszchuk K, Bernath PF, Zobov NF, Shirin SV, Polyansky OL, Libeskind NI, et al. Laboratory spectroscopy of hot water near 2-microns and sunspot spectroscopy in the H-band region. Astrophys J 2002;577:496-500.

[60] Sonnabend G, Wirtz D, Schieder R, Bernath P. High-resolution infrared measurements of $\mathrm{H}_{2} \mathrm{O}$ and $\mathrm{SiO}$ in sunspots. Solar Phys 2006;233:205-13. doi:10. 1007/s11207-006-2488-9.

[61] Polyansky OL, Kyuberis AA, Zobov NF, Tennyson J, Yurchenko SN, Lodi L. ExoMol molecular line lists XXX: a complete high-accuracy line list for water. Mon Not R Astron Soc 2018;480:2597-608. doi:10.1093/mnras/sty1877.

[62] Tennyson J, Yurchenko SN, Al-Refaie AF, Barton EJ, Chubb KL, Coles PA, et al. The ExoMol database: molecular line lists for exoplanet and other hot atmospheres. J Mol Spectrosc 2016;327:73-94. doi:10.1016/j.jms.2016.05.002.

[63] Ito Y, Ikoma M, Kawahara H, Nagahara H, Kawashima Y, Nakamoto T. Theoretical emission spectra of atmospheres of hot rocky super-earths. Astrophys J 2015;801:144. doi:10.1088/0004-637X/801/2/144.

[64] Fegley JB, Jacobson NS, Williams KB, Plane JMC, Schaefer L, Lodders K. Solubility of rock in steam atmospheres of planets. Astrophys J 2016;824:103. doi:10.3847/0004-637X/824/2/103

[65] Tennyson J, Yurchenko SN. Laboratory spectra of hot molecules: data needs for hot super-earth exoplanets. Mol Astrophys 2017;8:1-18. doi:10.1016/j. molap.2017.05.002.

[66] Herbort O, Woitke P, Helling C, Zerkle A. The atmospheres of rocky exoplanets I. outgassing of common rock and the stability of liquid water. Astron Astrophys 2020;636:A71. doi:10.1051/0004-6361/201936614.

[67] Gaudi BS, Stassun KG, Collins KA, Beatty TG, Zhou G, Latham DW, et al. A giant planet undergoing extreme-ultraviolet irradiation by its hot massivestar host. Nature 2017;546:514+. doi:10.1038/nature22392.

[68] Snellen I. High-dispersion spectroscopy of extrasolar planets: from CO in hot jupiters to $\mathrm{O}_{2}$ in exo-earths. Phil Trans Royal Soc London A 2014:372:20130075. doi:10.1098/rsta.2013.0075.

[69] Birkby JL. Spectroscopic direct detection of exoplanets. Handbook of Exoplanets 2018:1485-508.

[70] Hoeijmakers HJ, de Kok RJ, Snellen IAG, Brogi M, Birkby JL, Schwarz H. A search for $\mathrm{TiO}$ in the optical high-resolution transmission spectrum of HD 209458b: hindrance due to inaccuracies in the line database. Astron Astrophys 2015;575:A20. doi:10.1051/0004-6361/201424794.

[71] Rothman LS, Jacquemart D, Barbe A, Benner DC, Birk M, Brown LR, et al. The HITRAN 2004 molecular spectroscopic database. J Quant Spectrosc Radiat Transf 2005;96:139-204.

[72] Rothman LS, Gordon IE, Barbe A, Benner DC, Bernath PF, Birk M, et al. The HITRAN 2008 molecular spectroscopic database. J Quant Spectrosc Radiat Transf 2009;110:533-72.

[73] Down MJ, Hill C, Yurchenko SN, Tennyson J, Brown LR, Kleiner I. Re-analysis of ammonia spectra: updating the HITRAN ${ }^{14} \mathrm{NH}_{3}$ database. J Quant Spectrosc Radiat Transf 2013;130:260-72. doi:10.1016/j.jqsrt.2013.05.027.

[74] Jacquinet-Husson N, Crepeau L, Armante R, Boutammine C, Chédin A, Scott NA, et al. The 2009 edition of the GEISA spectroscopic database. J Quant Spectrosc Radiat Transf 2011;112:2395-445.

[75] Jacquinet-Husson N, Armante R, Scott NA, Chédin A, Crépeau L, Boutammine $C$, et al. The 2015 edition of the GEISA spectroscopic database. J Mol Spectrosc 2016;327:31-72. doi:10.1016/j.jms.2016.06.007.

[76] Rothman LS, Gordon IE, Barber RJ, Dothe H, Gamache RR, Goldman A, et al. HITEMP, The high-temperature molecular spectroscopic database. J Quant Spectrosc Radiat Transf 2010;111:2139-50.

[77] Hargreaves RJ, Gordon IE, Rothman LS, Tashkun SA, Perevalov VI, Lukashevskaya AA, et al. Spectroscopic line parameters of $\mathrm{NO}, \mathrm{NO}_{2}$, and $\mathrm{N}_{2} \mathrm{O}$ for the HITEMP database. J Quant Spectrosc Radiat Transf 2019;232:35-53. doi:10.1016/j.jqsrt.2019.04.040.

[78] Hargreaves RJ, Gordon IE, Rey M, Nikitin AV, Tyuterev VG, Kochanov RV, et al. An accurate, extensive, and practical line list of methane for the HITEMP database. Astrophys J Suppl 2020;247:55.

[79] Conway EK, Gordon IE, Hargreaves RJ, Gamache RR, Polyansky OL, Tennyson J. An accurate high temperature water line list for the HITEMP database. J Quant Spectrosc Radiat Transf 2020; In Preparation.
[80] Rey M, Nikitin AV, Babikov YL, Tyuterev VG. TheoReTS-an information system for theoretical spectra based on variational predictions from molecular potential energy and dipole moment surfaces. J Mol Spectrosc 2016;327:13858. doi:10.1016/j.jms.2016.04.006.

[81] Kurucz RL. Including all the lines. Can J Phys 2011;89:417-28.

[82] Bernath PF. MoLLIST: molecular line lists, intensities and spectra. J Quant Spectrosc Radiat Transf 2020;240:106687. doi:10.1016/j.jqsrt.2019.106687.

[83] Wang Y, Tennyson J, Yurchenko SN. Empirical line lists in the ExoMol database. Atoms 2020;8:7. doi:10.3390/atoms8010007.

[84] Riviére P, Soufiani A. Updated band model parameters for $\mathrm{H}_{2} \mathrm{O}, \mathrm{CO}_{2}, \mathrm{CH}_{4}$ and CO radiation at high temperature. Intern J Heat Mass Transf 2012;55:3349 58. doi:10.1016/j.ijheatmasstransfer.2012.03.019.

[85] Parigger CG, Woods AC, Surmick DM, Gautam G, Witte MJ, Hornkohl J. Computation of diatomic molecular spectra for selected transitions of aluminum monoxide, cyanide, diatomic carbon, and titanium monoxide. Spectra Chimica Acta B 2015;107:132-8. doi:10.1016/j.sab.2015.02.018.

[86] Tennyson J. Accurate variational calculations for line lists to model the vibration rotation spectra of hot astrophysical atmospheres. WIREs Comput Mol Sci 2012;2:698-715. doi:10.1002/wcms.94

[87] Tennyson J. Vibration-rotation transition dipoles from first principles. J Mol Spectrosc 2014;298:1-6. doi:10.1016/j.jms.2014.01.012.

[88] Tennyson J, Hulme K, Naim OK, Yurchenko SN. Radiative lifetimes and cooling functions for astrophysically important molecules. J Phys B 2016;49:044002 doi:10.1088/0953-4075/49/4/044002.

[89] Tennyson J, Lodi L, McKemmish LK, Yurchenko SN. The ab initio calculation of spectra of open shell diatomic molecules. J Phys B 2016;49:102001.

[90] Tennyson J. Perspective: accurate ro-vibrational calculations on small molecules. J Chem Phys 2016;145:120901. doi:10.1063/1.4962907.

[91] Semenov M, Yurchenko SN, Tennyson J. Predicted Landé g-factors for open shell diatomic molecules. J Mol Spectrosc 2017;330:57-62. doi:10.1016/j.jms 2016.11.004.

[92] Yurchenko SN, Lodi L, Tennyson J, Stolyarov AV. Duo: a general program for calculating spectra of diatomic molecules. Comput Phys Commun 2016;202:262-75. doi:10.1016/j.cpc.2015.12.021.

[93] Tennyson J, Yurchenko SN. The ExoMol project: software for computing molecular line lists. Intern J Quantum Chem 2017;117:92-103. doi:10.1002/ qua.25190.

[94] Yadin B, Vaness T, Conti P, Hill C, Yurchenko SN, Tennyson J. ExoMol molecular linelists: $\mathrm{i}$ the rovibrational spectrum of $\mathrm{BeH}, \mathrm{MgH}$ and $\mathrm{CaH}$ the $x^{2} \Sigma^{+}$ state. Mon Not R Astron Soc 2012;425:34-43.

[95] Barton EJ, Yurchenko SN, Tennyson J. ExoMol molecular linelists-II. the ro-vibrational spectrum of sio. Mon Not R Astron Soc 2013:434:1469-75.

[96] Barber RJ, Strange JK, Hill C, Polyansky OL, Mellau GC, Yurchenko SN, et al. ExoMol line lists-III. an improved hot rotation-vibration line list for HCN and HNC. Mon Not R Astron Soc 2014;437:1828-35. doi:10.1093/mnras/stt2011.

[97] Yurchenko SN, Tennyson J. ExoMol line lists IV: the rotation-vibration spectrum of methane up to $1500 \mathrm{~K}$. Mon Not R Astron Soc 2014;440:1649-61.

[98] Yurchenko SN, Amundsen DS, Tennyson J, Waldmann IP. A hybrid line list for $\mathrm{CH}_{4}$ and hot methane continuum. Astron Astrophys 2017;605:A95. doi:10. 1051/0004-6361/201731026.

[99] Barton EJ, Chiu C, Golpayegani S, Yurchenko SN, Tennyson J, Frohman DJ, et al. ExoMol molecular linelists-V. the ro-vibrational spectra of $\mathrm{nNaCl}$ and $\mathrm{KCl}$. Mon Not R Astron Soc 2014;442:1821-9. doi:10.1093/mnras/stu944.

[100] Yorke L, Yurchenko SN, Lodi L, Tennyson J. ExoMol line lists VI: a high temperature line list for phosphorus nitride. Mon Not R Astron Soc 2014;445:138391. doi:10.1093/mnras/stu1854.

[101] Sousa-Silva C, Al-Refaie AF, Tennyson J, Yurchenko SN. Exomol line lists - VII. the rotation-vibration spectrum of phosphine up to $1500 \mathrm{k}$. Mon Not R Astron Soc 2015;446:2337-47. doi:10.1093/mnras/stu2246.

[102] Al-Refaie AF, Yurchenko SN, Yachmenev A, Tennyson J. ExoMol line lists - VIII: a variationally computed line list for hot formaldehyde. Mon Not R Astron Soc 2015;448:1704-14. doi:10.1093/mnras/stv091.

[103] Patrascu AT, Tennyson J, Yurchenko SN. ExoMol molecular linelists: VII: the spectrum of AlO. Mon Not R Astron Soc 2015;449:3613-19. doi:10.1093/ mnras/stv507.

[104] Rivlin T, Lodi L, Yurchenko SN, Tennyson J, Le Roy RJ. ExoMol line lists X: the spectrum of sodium hydride. Mon Not R Astron Soc 2015;451:5153-7. doi:10.1093/mnras/stv979.

[105] Pavlyuchko AI, Yurchenko SN, Tennyson J. ExoMol line lists XI: a hot line list for nitric acid. Mon Not R Astron Soc 2015;452:1702-6. doi:10.1093/mnras/ stv1376.

[106] Paulose G, Barton EJ, Yurchenko SN, Tennyson J. ExoMol molecular linelistsXII. line lists for eight isotopologues of CS. Mon Not R Astron Soc 2015;454:1931-9. doi:10.1093/mnras/stv1543.

[107] Yurchenko SN, Blissett A, Asari U, Vasilios M, Hill C, Tennyson J. ExoMol molecular linelists-XIII. the spectrum of $\mathrm{CaO}$. Mon Not R Astron Soc 2016;456:4524-32. doi:10.1093/mnras/stv2858.

[108] Underwood DS, Tennyson J, Yurchenko SN, Huang X, Schwenke DW, Lee TJ, et al. ExoMol line lists XIV: a line list for hot $\mathrm{SO}_{2}$. Mon Not $\mathrm{R}$ Astron Soc 2016;459:3890-9. doi:10.1093/mnras/stw849.

[109] Al-Refaie AF, Polyansky OL, Ovsyannikov RI, Tennyson J, Yurchenko SN. ExoMol line lists XV: a hot line-list for hydrogen peroxide. Mon Not R Astron Soc 2016;461:1012-22. doi:10.1093/mnras/stw1295.

[110] Azzam AAA, Yurchenko SN, Tennyson J, Naumenko OV. ExoMol line lists XVI: a hot line list for $\mathrm{H}_{2}$ S. Mon Not R Astron Soc 2016;460:4063-74. doi:10.1093/ mnras/stw1133. 
[111] Underwood DS, Tennyson J, Yurchenko SN, Clausen S, Fateev A. ExoMol line lists XVII: a line list for hot $\mathrm{SO}_{3}$. Mon Not R Astron Soc 2016;462:4300-13. doi:10.1093/mnras/stw1828.

[112] McKemmish LK, Yurchenko SN, Tennyson J. ExoMol molecular linelists-XVIII. the spectrum of vanadium oxide. Mon Not R Astron Soc 2016;463:771-93. doi:10.1093/mnras/stw1969.

[113] Polyansky OL, Kyuberis AA, Lodi L, Tennyson J, Ovsyannikov RI, Zobov N. ExoMol molecular line lists XIX: high accuracy computed line lists for $\mathrm{H}_{2}{ }^{17} \mathrm{O}$ and $\mathrm{H}_{2}{ }^{18} \mathrm{O}$. Mon Not R Astron Soc 2017;466:1363-71. doi:10.1093/mnras/stw3125.

[114] Mizus II, Alijah A, Zobov NF, Kyuberis AA, Yurchenko SN, Tennyson J, et al. ExoMol molecular line lists XX: a comprehensive line list for $\mathrm{H}_{3}^{+}$. Mon Not $\mathrm{R}$ Astron Soc 2017;468:1717-25. doi:10.1093/mnras/stx502.

[115] Wong A, Yurchenko SN, Bernath P, Mueller HSP, McConkey S, Tennyson J. ExoMol line list XXI: nitric oxide (NO). Mon Not R Astron Soc 2017;470:882-97. doi:10.1093/mnras/stx1211.

[116] Owens A, Yurchenko SN, Yachmenev A, Thiel W, Tennyson J. ExoMol molecular line lists XXII. the rotation-vibration spectrum of silane up to $1200 \mathrm{~K}$. Mon Not R Astron Soc 2017;471:5025-32. doi:10.1093/mnras/stx1952.

[117] Prajapat L, Jagoda P, Lodi L, Gorman MN, Yurchenko SN, Tennyson J. ExoMol molecular line lists XXIII. spectra of PO and PS. Mon Not R Astron Soc 2017:472:3648-58. doi:10.1093/mnras/stx2229.

[118] Yurchenko SN, Sinden F, Lodi L, Hill C, Gorman MN, Tennyson J. ExoMol molecular linelists-XXIV: a new hot line list for silicon monohydride, $\mathrm{SiH}$. Mon Not R Astron Soc 2018;473:5324-33. doi:10.1093/mnras/stx2738.

[119] Upadhyay A, Conway EK, Tennyson J, Yurchenko SN. ExoMol molecular linelists-XXV: a hot line list for silicon sulphide, SiS. Mon Not R Astron Soc 2018;477:1520-7. doi:10.1093/mnras/sty998.

[120] Yurchenko SN, Bond W, Gorman MN, Lodi L, McKemmish LK, Nunn W, et al. ExoMol molecular linelists-XXVI: spectra of SH and NS. Mon Not R Astron Soc 2018;478:270-82. doi:10.1093/mnras/sty939.

[121] Mant BP, Yachmenev A, Tennyson J, Yurchenko SN. ExoMol molecular line lists - XXVII: spectra of $\mathrm{C}_{2} \mathrm{H}_{4}$. Mon Not R Astron Soc 2018;478:3220-32. doi:10. 1093/mnras/sty1239.

[122] Yurchenko SN, Williams H, Leyland PC, Lodi L, Tennyson J. ExoMol line lists XXVIII: the rovibronic spectrum of AlH. Mon Not $\mathrm{R}$ Astron Soc 2018;479:1401-11. doi:10.1093/mnras/sty1524.

[123] Owens A, Yachmenev A, Tennyson J, Thiel W, Yurchenko SN. ExoMol molecular line lists XXIX: the rotation-vibration spectrum of methyl chloride up to 1200 K. Mon Not R Astron Soc 2018;479:3002-10. doi:10.1093/mnras/ sty1542.

[124] Yurchenko SN, Szabo I, Pyatenko E, Tennyson J. ExoMol molecular line lists XXXI: the spectrum of $C_{2}$. Mon Not R Astron Soc 2018;480:3397-411. doi:10. 1093/mnras/sty2050.

[125] Li HY, Tennyson J, Yurchenko SN. ExoMol molecular line lists XXXII: the rovibronic spectrum of MgO. Mon Not R Astron Soc 2019;486:2351-65. doi:10. 1093/mnras/stz912

[126] McKemmish LK, Masseron T, Hoeijmakers J, Pérez-Mesa VV, Grimm SL, Yurchenko SN, et al. ExoMol molecular line lists-XXXIII. The spectrum of titanium oxide. Mon Not R Astron Soc 2019;488:2836-54.

[127] Langleben J, Yurchenko SN, Tennyson J. ExoMol line list XXXIV: a rovibrational line list for phosphinidene (PH) in its $x^{3} \Sigma^{-}$and $a^{1} \Delta$ electronic states. Mon Not R Astron Soc 2019;488:2332. doi:10.1093/mnras/stz1856-2342.

[128] Coles PA, Yurchenko SN, Tennyson J. ExoMol molecular line lists XXXV: a rotation-vibration line list for hot ammonia. Mon Not $\mathrm{R}$ Astron Soc 2019;490:4638-47. doi:10.1093/mnras/stz2778.

[129] Gorman M, Yurchenko SN, Tennyson J. ExoMol molecular linelists-XXXVI. $x^{2} \Pi-x^{2} \Pi$ and $a^{2} \Sigma^{+}-x^{2} \Pi$ transitions of SH. Mon Not $\mathrm{R}$ Astron Soc 2019;490:1652-65. doi:10.1093/mnras/stz2517/5565070.

[130] Chubb KL, Tennyson J, Yurchenko SN. ExoMol molecular linelists-XXXVII: spectra of acetylene. Mon Not R Astron Soc 2020;493:1531-45. doi:10.1093/ mnras/staa229.

[131] Owens A, Conway EK, Tennyson J, Yurchenko SN. ExoMol molecular linelistsXXXVIII: high-temperature molecular line list of silicon dioxide $\left(\mathrm{SiO}_{2}\right)$. Mon Not R Astron Soc 2020;495:1927-33. doi:10.1093/mnras/staa1287.

[132] Yurchenko SN, Mellor TM, Freedman RS, Tennyson J. ExoMol molecular line lists XXXIX: ro-vibrational molecular line list for $\mathrm{CO}_{2}$. Mon Not R Astron Soc 2020. doi:10.1093/mnras/staa 1874.

[133] Yurchenko SN, Tennyson J, Miller S, Melnikov VV, O’Donoghue J, Moore L. ExoMol molecular line lists XL: ro-vibrational molecular line list for the hydronium ion $\left(\mathrm{H}_{3} \mathrm{O}^{+}\right)$. Mon Not R Astron Soc 2020.

[134] Harris GJ, Larner FC, Tennyson J, Kaminsky BM, Pavlenko YV, Jones HRA. A $\mathrm{H}^{13} \mathrm{CN} / \mathrm{HN}^{13} \mathrm{C}$ linelist, model atmospheres and synthetic spectra for carbon stars. Mon Not R Astron Soc 2008:390:143-8.

[135] Sochi T, Tennyson J. A computed line list for the $\mathrm{H}_{2} \mathrm{D}^{+}$molecular ion. Mon Not R Astron Soc 2010;405:2345-50.

[136] Voronin BA, Tennyson J, Tolchenov RN, Lugovskoy AA, Yurchenko SN. A high accuracy computed line list for the HDO molecule. Mon Not R Astron Soc 2010;402:492-6

[137] Yurchenko SN. A theoretical room-temperature line list for ${ }^{15} \mathrm{NH}_{3}$. J Quant Spectrosc Radiat Transf 2015;152:28-36. doi:10.1016/j.jqsrt.2014.10.023.

[138] Roueff E, Abgrall H, Czachorowski P, Pachucki K, Puchalski M, Komasa J. The full infrared spectrum of molecular hydrogen. Astron Astrophys 2019;630:A58. doi:10.1051/0004-6361/201936249.

[139] Masseron T, Plez B, Van Eck S, Colin R, Daoutidis I, Godefroid M, et al. CH in stellar atmospheres: an extensive linelist. Astron Astrophys 2014;571:A47. doi:10.1051/0004-6361/201423956.
[140] Brooke JSA, Bernath PF, Western CM, van Hemert MC, Groenenboom GC. Line strengths of rovibrational and rotational transitions within the $\mathrm{X} \Sigma_{3}{ }^{-}$ground state of NH. J Chem Phys 2014;141:054310. doi:10.1063/1.4891468.

[141] Brooke JSA, Bernath PF, Western CM. Note: improved line strengths of rovi-

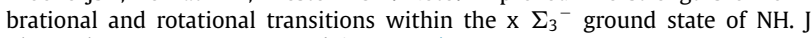
Chem Phys 2015;143:026101. doi:10.1063/1.4923422.

[142] Fernando AM, Bernath PF, Hodges JN, Masseron T. A new linelist for the a ${ }^{3} \Pi-\mathrm{x} \Sigma_{3}{ }^{-}$transition of the NH free radical. J Quant Spectrosc Radiat Transf 2018;217:29-34. doi:10.1016/j.jqsrt.2018.05.021.

[143] Brooke JSA, Bernath PF, Western CM, Sneden C, Afsar M, Li G, et al. Line strengths of rovibrational and rotational transitions in the $X^{2} \Pi$ ground state of OH. J Quant Spectrosc Radiat Transf 2016;138:142-57. doi:10.1016/j.jqsrt. 2015.07.021.

[144] Yousefi M, Bernath PF. Line lists for AlF and $\mathrm{AlCl}$ in the $x^{1} \Sigma^{+}$ground state. Astrophys J Suppl 2018;237:8. doi:10.3847/1538-4365/aacc6a.

[145] Hodges JN, Bernath PF. Fourier transform spectroscopy of the $a^{3} \Pi-x^{3} \Sigma^{-}$ transition of $\mathrm{OH}^{+}$. Astrophys J 2017;840:81. doi:10.3847/1538-4357/aa6bf5.

[146] Hodges JN, Bittner DM, Bernath PF. Improved ultraviolet and infrared oscillator strengths for $\mathrm{OH}^{+}$. Astrophys J 2018;855. doi:10.3847/1538-4357/aaa7ef.

[147] Hou S, Bernath PF. Line list for the ground state of CaF. J Quant Spectrosc Radiat Transf 2018;210:44-51. doi:10.1016/j.jqsrt.2018.02.011.

[148] Hou S, Bernath PF. Line list for the MgF ground state. J Quant Spectrosc Radiat Transf 2017;203:511-16.

[149] Frohman DJ, Bernath PF, Brooke JSA. Molecular line lists: the ro-vibrational spectra of NaF and KF. J Quant Spectrosc Radiat Transf 2016;169:104-10. doi:10.1016/j.jqsrt.2015.10.004

[150] Bittner DM, Bernath PF. Line lists for LiF and LiCl in the $\mathrm{x}^{1} \Sigma^{+}$ground state. Astrophys J Suppl 2018;235:8. doi:10.3847/1538-4365/aa9846.

[151] GharibNezhad E, Shayesteh A, Bernath PF. Einstein A coefficients for rovibronic lines of the $A^{2} \Pi \rightarrow X^{2} \Sigma^{+}$and $B^{\prime 2} \Sigma^{+} \rightarrow X^{2} \Sigma^{+}$transitions of mgh. Mon Not R Astron Soc 2013;432:2043-7. doi:10.1093/mnras/stt510.

[152] Burrows A, Dulick M, Bauschlicher CW, Bernath PF, Ram RS, Sharp CM, et al. Spectroscopic constants, abundances, and opacities of the TiH molecule. Astrophys J 2005;624:988-1002. doi:10.1086/429366.

[153] Chowdhury PK, Merer AJ, Rixon SJ, Bernath PF, Ram RS. Low-N lines of the a $\Sigma_{6}{ }^{+}-\mathrm{x} \Sigma_{6}{ }^{+}(1,0)$ band of CrH. Phys Chem Chem Phys 2006;8:822-6. doi:10. 1039/b514188e.

[154] Wende S, Reiners A, Seifahrt A, Bernath PF. Crires spectroscopy and empirical line-by-line identification of $\mathrm{FeH}$ molecular absorption in an M dwarf. Astron Astrophys 2010;523:A58. doi:10.1051/0004-6361/201015220.

[155] Coxon JA, Hajigeorgiou PG. Improved direct potential fit analyses for the ground electronic states of the hydrogen halides: $\mathrm{HF} / \mathrm{DF} / \mathrm{TF}, \mathrm{HCl} / \mathrm{DCl} / \mathrm{TCl}$, $\mathrm{HBr} / \mathrm{DBr} / \mathrm{TBr}$ and HI/DI/TI. J Quant Spectrosc Radiat Transf 2015;151:133-54. doi:10.1016/j.jqsrt.2014.08.028.

[156] Li G, Gordon IE, Bernath PF, Rothman LS. Direct fit of experimental rovibrational intensities to the dipole moment function: application to $\mathrm{HCl}$. J Quant Spectrosc Radiat Transf 2011;112:1543-50. doi:10.1016/j.jqsrt.2011.03. 014.

[157] Ram RS, Brooke JSA, Western CM, Bernath PF. Einstein a-values and oscillator strengths of the a ${ }^{2} \Pi-x \Sigma_{2}{ }^{+}$system of CP. J Quant Spectrosc Radiat Transf 2014;138:107-15. doi:10.1016/j.jqsrt.2014.01.030.

[158] Brooke JSA, Ram RS, Western CM, Li G, Schwenke DW, Bernath PF. Einstein A coefficients and oscillator strengths for the $\mathrm{A}^{2} \Pi-\mathrm{X}^{2} \Sigma^{+}$(red) and $\mathrm{B}^{2} \Sigma^{+}$$\mathrm{X}^{2} \Sigma^{+}$violet systems and rovibrational transitions in the $\mathrm{X}^{2} \Sigma^{+}$state of $\mathrm{CN}$. Astrophys J Suppl 2014;210:23. doi:10.1088/0067-0049/210/2/23.

[159] Brooke JSA, Bernath PF, Schmidt TW, Bacskay GB. Line strengths and updated molecular constants for the $C_{2}$ Swan system. J Quant Spectrosc Radiat Transf 2013;124:11-20. doi:10.1016/j.jqsrt.2013.02.025

[160] Li G, Harrison JJ, Ram RS, Western CM, Bernath PF. Einstein A coefficients and absolute line intensities for the $\mathrm{E}^{2} \Pi-\mathrm{X} \Sigma_{2}{ }^{+}$transition of $\mathrm{CaH}$. J Quant Spectrosc Radiat Transf 2012;113:67-74. doi:10.1016/j.jqsrt.2011.09.010.

[161] Shayesteh A, Ram RS, Bernath PF. Fourier transform emission spectra of the $A^{2} \Pi \rightarrow X^{2} \Sigma^{+}$and $B^{2} \Sigma^{+} \rightarrow X^{2} \Sigma^{+}$band systems of CaH. J Mol Spectrosc 2013;288:46-51. doi:10.1016/j.jms.2013.04.009.

[162] Western CM, Carter-Blatchford L, Crozet P, Ross AJ, Morville J, Tokaryk DW. The spectrum of $\mathrm{N}_{2}$ from 4,500 to $15,700 \mathrm{~cm}^{-1}$ revisited with PGOPHER. J Quant Spectrosc Radiat Transf 2018;219:127-41. doi:10.1016/j.jqsrt.2018.07. 017.

[163] Lodi L, Yurchenko SN, Tennyson J. The calculated rovibronic spectrum of scandium hydride, ScH. Mol Phys 2015;113:1559-75. doi:10.1080/00268976.2015. 1029996.

[164] Coppola CM, Lodi L, Tennyson J. Radiative cooling functions for primordial molecules. Mon Not R Astron Soc 2011;415:487-93.

[165] Li G, Gordon IE, Rothman LS, Tan Y, Hu SM, Kassi S, et al. Rovibrational line lists for nine isotopologues of the CO molecule in the $\mathrm{X}^{1} \Sigma^{+}$ground electronic state. Astrophys J Suppl 2015;216:15. doi:10.1088/0067-0049/216/1/15.

[166] Amaral PHR, Diniz LG, Jones KA, Stanke M, Alijah A, Adamowicz L, et al. Benchmark rovibrational linelists and einstein A-coefficients for the primordial molecules and isotopologues. Astrophys J 2019;878(2):95. doi:10.3847/ 1538-4357/ab1f65.

[167] Owens A, Yachmenev A, Küpper J, Yurchenko SN, Thiel W. The rotationvibration spectrum of methyl fluoride from first principles. Phys Chem Chem Phys 2018;21:3496-505. doi:10.1039/С8CP01721B.

[168] Coles PA, Yurchenko SN, Kovacich RP, Hobby J, Tennyson J. A variationally computed room temperature line list for $\mathrm{AsH}_{3}$. Phys Chem Chem Phys 2019;21:3264-77. doi:10.1039/С8CP07110A. 
[169] Owens A, Yurchenko SN. Theoretical rotation-vibration spectroscopy of cisand trans-diphosphene $\left(\mathrm{P}_{2} \mathrm{H}_{2}\right)$ and the deuterated species $\mathrm{P}_{2} \mathrm{HD}$. J Chem Phys 2019;150(19):194308. doi:10.1063/1.5092767.

[170] Mant BP, Chubb KL, Yachmenev A, Tennyson J, Yurchenko SN. The infrared spectrum of $\mathrm{PF}_{3}$ and the analysis of rotational energy clustering effect. Mol Phys 2019;118:e1581951. doi:10.1080/00268976.2019.1581951.

[171] Adam AY, Yachmenev A, Yurchenko SN, Jensen P. A variationally computed IR line list for the methyl radical $\mathrm{CH}_{3}$. J Phys Chem A 2019;123:22. doi:10.1021/ acs.jpca.9b02919.

[172] Darby-Lewis D, Tennyson J, Lawson KD, Yurchenko SN, Stamp MF, Shaw A et al. Synthetic spectra of BeH, BeD and BeT for emission modelling in JET plasmas. J Phys B: At Mol Opt Phys 2018;51:185701. doi:10.1088/1361-6455/ aad6d0.

[173] Huang X, Freedman RS, Tashkun SA, Schwenke DW, Lee TJ. Semi-empirical ${ }^{12} \mathrm{C}^{16} \mathrm{O}_{2}$ IR line lists for simulations up to $1500 \mathrm{~K}$ and $20,000 \mathrm{~cm}^{-1}$. J Quant Spectrosc Radiat Transf 2013;130:134-46. doi:10.1016/j.jqsrt.2013.05.018.

[174] Huang X, Schwenke DW, Freedman RS, Lee TJ. Ames-2016 line lists for 13 isotopologues of $\mathrm{CO}_{2}$ : updates, consistency, and remaining issues. J Quant Spectrosc Radiat Transf 2017;203:224-41. doi:10.1016/j.jqsrt.2017.04.026.

[175] Clark VHJ, Owens A, Tennyson J, Yurchenko SN. The high-temperature rotation-vibration spectrum and rotational clustering of silylene $\left(\mathrm{SiH}_{2}\right)$. J Quant Spectrosc Radiat Transf 2020;246:106929. doi:10.1016/j.jqsrt.2020. 106929.

[176] Yurchenko SN, Smirnov AN, Solomonik VG, Tennyson J. Spectroscopy of YO from first principles. Phys Chem Chem Phys 2019;21:22794-810. doi:10.1039/ $\mathrm{C9CP03208H.}$

[177] Neale L, Miller S, Tennyson J. Spectroscopic properties of the $\mathrm{H}_{3}^{+}$molecule: a new calculated linelist. Astrophys J 1996;464:516-20.

[178] Barber RJ, Tennyson J, Harris GJ, Tolchenov RN. A high accuracy computed water line list. Mon Not R Astron Soc 2006;368:1087-94.

[179] Yurchenko SN, Barber RJ, Tennyson J. A variationally computed hot line list for $\mathrm{NH}_{3}$. Mon Not R Astron Soc 2011;413:1828-34. doi:10.1111/j.1365-2966. 2011.18261.x.

[180] Engel EA, Doss N, Harris GJ, Tennyson J. An opacity for $\mathrm{HeH}^{+}$and its effect on models of cool, metal poor stars. Mon Not R Astron Soc 2005;357:471-7.

[181] Zak EJ, Tennyson J, Polyansky OL, Lodi L, Tashkun SA, Perevalov VI. A room temperature $\mathrm{CO}_{2}$ line list with $a b$ initio computed intensities. J Quant Spectrosc Radiat Transf 2016;177:31-42. doi:10.1016/j.jqsrt.2015.12.022.

[182] Zak EJ, Tennyson J, Polyansky OL, Lodi L, Zobov NF, Tashkun SA, et al. Room temperature line lists for $\mathrm{CO}_{2}$ symmetric isotopologues with $a b$ initio computed intensities. J Quant Spectrosc Radiat Transf 2017;189:267-80. doi:10.1016/j.jqsrt.2016.11.022.

[183] Zak EJ, Tennyson J, Polyansky OL, Lodi L, Zobov NF, Tashkun SA, et al. Room temperature line lists for $\mathrm{CO}_{2}$ asymmetric isotopologues with $a b$ initio computed intensities. J Quant Spectrosc Radiat Transf 2017;203:265-81. doi:10.1016/j.jqsrt.2017.01.037.

[184] Tashkun SA, Perevalov VI. CDSD-4000: High-resolution, high-temperature carbon dioxide spectroscopic databank. J Quant Spectrosc Radiat Transf 2011;112:1403-10. doi:10.1016/j.jqsrt.2011.03.005.

[185] Conway EK, Gordon IE, Tennyson J, Polyansky OL, Yurchenko SN, Chance K. A semi-empirical potential energy surface and line list for $\mathrm{H}_{2}{ }^{16} \mathrm{O}$ extending into the near-ultraviolet. Atmos Chem Phys 2020.

[186] Medvedev ES, Meshkov VV, Stolyarov AV, Gordon IE. Peculiarities of high-overtone transition probabilities in carbon monoxide. J Chem Phys 2015;143:154301. doi:10.1063/1.4933136.

[187] Le Roy RJ. Level: a computer program for solving the radial Schrödinger equation for bound and quasibound levels. J Quant Spectrosc Radiat Transf 2017; 186:167-78. doi:10.1016/j.jqsrt.2016.05.028.

[188] Al Derzi AR, Furtenbacher T, Yurchenko SN, Tennyson J, Császár AG. MARVEL Analysis of the measured high-resolution spectra of ${ }^{14} \mathrm{NH}_{3}$. J Quant Spectrosc Radiat Transf 2015;161:117-30. doi:10.1016/j.jqsrt.2015.03.034.

[189] Medvedev ES, Ushakov VG, Conway EK, Upadhyay A, Gordon IE, Tennyson J. Empirical normal intensity distribution law for overtone vibrational spectra of triatomic molecules. J Quant Spectrosc Radiat Transf 2020;252:107084. doi:10.1016/j.jqsrt.2020.107084.

[190] Furtenbacher T, Császár AG, Tennyson J. MARVEL: Measured active rotationalvibrational energy levels. J Mol Spectrosc 2007;245:115-25. doi:10.1016/j.jms. 2007.07.005

[191] von Essen C, Mallonn M, Welbanks L, Madhusudhan N, Pinhas A, Bouy H, et al. An optical transmission spectrum of the ultra-hot Jupiter WASP-33 b first indication of aluminum oxide in an exoplanet. Astron Astrophys 2019;622:A71. doi:10.1051/0004-6361/201833837.

[192] Chubb K.L., Min M., Kawashima Y., Helling C., Waldmann I.. Aluminium oxide in the atmosphere of hot Jupiter WASP-43b. 2020b. Astron. Astrophys. 639, A3.

[193] Furtenbacher T, Szabó I, Császár AG, Bernath PF, Yurchenko SN, Tennyson J. Experimental energy levels and partition function of the ${ }^{12} \mathrm{c}_{2}$ molecule. Astrophys J Suppl 2016;224:44. doi:10.3847/0067-0049/224/2/44.

[194] McKemmish LK, Syme AM, Borsovszky J, Yurchenko SN, Tennyson J, Furtenbacher $\mathrm{T}$, et al. Incorportating new experiments in diatomic spectral databases: an update to the ${ }^{12} \mathrm{C}_{2}$ MARVEL database and exomok line list of ${ }^{12} C_{2}$. Mon Not R Astron Soc 2020.

[195] Ram RS, Tereszchuk K, Gordon IE, Walker KA, Bernath PF. Fourier transform emission spectroscopy of the $e^{2} \Pi-x^{2} \Sigma^{+}$transition of CaH and CaD. J Mol Spectrosc 2011;266:86-91. doi:10.1016/j.jms.2011.03.009.
[196] Hou S, Wei Z. Line lists for the $X^{1} \Sigma^{+}$state of CS. Astrophys J Suppl 2020;246:14. doi:10.3847/1538-4365/ab61 ef.

[197] Xing W, Shi D, Sun J. Rovibrational transition properties of the $\mathrm{x} \Sigma_{1}{ }^{+}$and a ${ }^{1} \Pi$ states of carbon monosulfide. Mol Phys 2020;0:1-13. doi:10.1080/ 00268976.2020.1759831.

[198] Pavlenko YV, Yurchenko SN, Tennyson J. Analysis of first overtone bands of isotopologues of $\mathrm{CO}$ and sio in stellar spectra. Astron Astrophys 2020;633:A52.

[199] McKemmish LK, Masseron T, Sheppard S, Sandeman E, Schofield Z, Furtenbacher T, et al. MARVEL Analysis of the measured high-resolution spectra of ${ }^{48} \mathrm{Ti}^{16}$ O. Astrophys J Suppl 2017;228:15. doi:10.3847/1538-4365/228/2/15.

[200] Sedaghati E, Boffin HMJ, MacDonald RJ, Gandhi S, Madhusudhan N, Gibson NP, et al. Detection of titanium oxide in the atmosphere of a hot Jupiter. Nature 2017;549:238+. doi:10.1038/nature23651.

[201] Nugroho SK, Kawahara H, Masuda K, Hirano T, Kotani T, Tajitsu A. Highresolution spectroscopic detection of $\mathrm{TiO}$ and a stratosphere in the day-side of WASP-33b. Astrophys J 2017;154:221. doi:10.3847/1538-3881/aa9433.

[202] Plez B. A new TiO line list. Astron Astrophys 1998;337:495-500.

[203] Schwenke DW. Opacity of TiO from a coupled electronic state calculation parametrized by $\mathrm{ab}$ initio and experimental data. Faraday Discuss 1998;109:321-34. doi:10.1039/a800070k.

[204] McKemmish LK, Yurchenko SN, Tennyson J. Ab initio calculations to support accurate modelling of the rovibronic spectroscopy calculations of vanadium monoxide (VO). Mol Phys 2016;114:3232-48. doi:10.1080/00268976. 2016.1225994.

[205] Evans TM, Sing DK, Wakeford HR, Nikolov N, Ballester GE, Drummond B, et al. Detection of $\mathrm{H}_{2} \mathrm{O}$ and evidence for TiO/VO in an ultra-hot exoplanet atmosphere. Astrophys J 2016;822:L4. doi:10.3847/2041-8205/822/1/14.

[206] Tsiaras A, Waldmann IP, Zingales T, Rocchetto M, Morello G, Damiano M, et al. A population study of gaseous exoplanets. Astron J 2018;155:156 doi:10.3847/1538-3881/aaaf75.

[207] Cheung ASC, Hansen RC, Merer AJ. Laser spectroscopy of VO - analysis of the rotational and hyperfine structure of the $C^{4} \Sigma-X^{4} \Sigma-(0,0)$ band. J Mol Spectrosc 1982;91:165-208. doi:10.1016/0022-2852(82)90039-X.

[208] Polyansky OL, Bielska K, Ghysels M, Lodi L, Zobov NF, Hodges JT, et al. High accuracy $\mathrm{CO}_{2}$ line intensities determined from theory and experiment. Phys Rev Lett 2015;114:243001. doi:10.1103/PhysRevLett.114.243001.

[209] Huang X, Schwenke DW, Freedman RS, Lee T]. Ames-2016 line lists for 13 isotopologues of $\mathrm{CO}_{2}$ : updates, consistency, and remaining issues. J Quant Spectrosc Radiat Transf 2017;203:224-41. doi:10.1016/j.jqsrt.2017.04.026.

[210] Mellau GC. Complete experimental rovibrational eigenenergies of HCN up to $6880 \mathrm{~cm}^{-1}$ above the ground state. J Chem Phys 2011:134:234303.

[211] Mellau GC. Highly excited rovibrational states of HNC. J Mol Spectrosc 2011;269:77-85,

[212] Hawker GA, Madhusudhan N, Cabot SHC, Gandhi S. Evidence for multiple molecular species in the hot Jupiter HD 209458b. Astrophys J Lett 2018;863:L11. doi:10.3847/2041-8213/aac49d.

[213] Cabot SHC, Madhusudhan N, Hawker GA, Gandhi S. On the robustness of analysis techniques for molecular detections using high-resolution exoplanet spectroscopy. Mon Not R Astron Soc 2019;482:4422-36. doi:10.1093/mnras/ sty2994.

[214] Gandhi S, Brogi M, Yurchenko SN, Tennyson J, Coles PA, Webb RK, et al. Molecular cross sections for high resolution spectroscopy of super earths, warm neptunes and hot Jupiters. Mon Not R Astron Soc 2020;495:224-37. doi:10.1093/mnras/staa981.

[215] Tsiaras A, Waldmann IP, Tinetti G, Tennyson J, Yurchenko SN. Water vapour in the atmosphere of the habitable-zone eight-earth-mass planet K2 18b. Nature Astron 2019;3:1086-91. doi:10.1038/s41550-019-0878-9.

[216] Partridge $\mathrm{H}$, Schwenke DW. The determination of an accurate isotope dependent potential energy surface for water from extensive ab initio calculations and experimental data. J Chem Phys 1997;106:4618-39. doi:10.1063/1.473987.

[217] Tennyson J, Bernath PF, Brown LR, Campargue A, Carleer MR, Császár AG, et al. IUPAC Critical evaluation of the rotational-vibrational spectra of water vapor. part III. energy levels and transition wavenumbers for $\mathrm{H}_{2}{ }^{16} \mathrm{O}$. J Quant Spectrosc Radiat Transf 2013;117:29-80. doi:10.1016/j.jqsrt.2012.10.002.

[218] Lampel J, Pöhler D, Polyansky OL, Kyuberis AA, Zobov NF, Tennyson J, et al. Detection of water vapour absorption around $363 \mathrm{~nm}$ in measured atmospheric absorption spectra and its effect on DOAS evaluations. Atmos Chem Phys 2017;17:1271-95. doi:10.5194/acp-2016-388.

[219] Lodi L, Tennyson J, Polyansky OL. A global, high accuracy ab initio dipole moment surface for the electronic ground state of the water molecule. J Chem Phys 2011;135:034113. doi:10.1063/1.3604934.

[220] Conway EK, Kyuberis AA, Polyansky OL, Tennyson J, Zobov N. A highly accurate $a b$ initio dipole moment surface for the ground electronic state of water vapour for spectra extending into the ultraviolet. J Chem Phys 2018;149:084307. doi:10.1063/1.5043545.

[221] Conway EK, Gordon IE, Kyuberis AA, Polyansky OL, Tennyson J, Zobov NF. Accurate line lists for $\mathrm{H}_{2}{ }^{16} \mathrm{O}, \mathrm{H}_{2}{ }^{18} \mathrm{O}$ and $\mathrm{H}_{2}{ }^{17} \mathrm{O}$ with extensive comparisons to theoretical and experimental sources including the HITRAN2016 database. J Quant Spectrosc Radiat Transf 2020;241:106711. doi:10.1016/j.jqsrt.2019. 106711.

[222] Conway EK, Gordon IE, Polyansky OL, Tennyson J. Use of the complete basis set limit for computing highly accurate $a b$ initio dipole moments. J Chem Phys 2020;152:024105. doi:10.1063/1.5135931.

[223] Tennyson J, Bernath PF, Brown LR, Campargue A, Carleer MR, Császár AG, et al. IUPAC Critical evaluation of the rotational-vibrational spectra of water 
vapor. part I. energy levels and transition wavenumbers for $\mathrm{H}_{2}{ }^{17} \mathrm{O}$ and $\mathrm{H}_{2}{ }^{18} \mathrm{O}$. J Quant Spectrosc Radiat Transf 2009;110:573-96. doi:10.1016/j.jqsrt.2009.02. 014.

[224] Tennyson J, Bernath PF, Brown LR, Campargue A, Carleer MR, Császár AG, et al. IUPAC Critical evaluation of the rotational-vibrational spectra of water vapor. part II. energy levels and transition wavenumbers for $\operatorname{HD}^{16} \mathrm{O}, \mathrm{HD}^{17} \mathrm{O}$, and HD ${ }^{18}$ O. J Quant Spectrosc Radiat Transf 2010;111:2160-84. doi:10.1016/j. jqsit.2010.06.012.

[225] Zobov NF, Koshelev MA, Makaro DS, Boyarkin OV, Tyuterev VG, Tennyson J, et al. Global study of the HDO spectrum - analysis of experiment and high temperature line list. J Quant Spectrosc Radiat Transf 2020; In Preparation.

[226] Campargue A, Kassi S, Yachmenev A, Kyuberis AA, Küpper J, Yurchenko SN. Observation of electric-quadrupole infrared transitions in water vapor. Phys Rev Research 2020;2:023091. doi:10.1103/PhysRevResearch.2.023091.

[227] Mizus II, Kyuberis AA, Zobov NF, Makhnev VY, Polyansky OL, Tennyson J. High accuracy water potential energy surface for the calculation of infrared spectra. Phil Trans Royal Soc London A 2018;376:20170149. doi:10.1098/rsta.2017. 0149.

[228] Yurchenko SN, Thiel W, Jensen P. Theoretical ROVibrational Energies (TROVE): a robust numerical approach to the calculation of rovibrational energies for polyatomic molecules. J Mol Spectrosc 2007;245:126-40. doi:10.1016/j.jms. 2007.07.009.

[229] Gordon IE, Rothman LS, Hill C, Kochanov RV, Tan Y, Bernath PF, et al. The HITRAN 2016 molecular spectroscopic database. J Quant Spectrosc Radiat Transf 2017;203:3-69. doi:10.1016/j.jqsrt.2017.06.038.

[230] Chubb KL, Naumenko OV, Keely S, Bartolotto S, MacDonald S, Mukhtar M, et al. MARVEL Analysis of the measured high-resolution rovibrational spectra of $\mathrm{H}_{2} \mathrm{~S}$. J Quant Spectrosc Radiat Transf 2018;218:178-86. doi:10.1016/j.jqsrt. 2018.07.012.

[231] Furtenbacher T, Szidarovszky T, Fábri C, Császár AG. MARVEL Analysis of the rotational-vibrational states of the molecular ions $\mathrm{H}_{2} \mathrm{D}^{+}$and $\mathrm{D}_{2} \mathrm{H}^{+}$. Phys Chem Chem Phys 2013;15:10181-93. doi:10.1039/c3cp44610g.

[232] Petrignani A, Berg M, Wolf A, Mizus II, Polyansky OL, Tennyson J, et al. Visible intensities of the triatomic hydrogen ion from experiment and theory. J Chem Phys 2014;141:241104. doi:10.1063/1.4904440.

[233] Miller S, Geballe TR, Stallard T, Tennyson J. Thirty years of $\mathrm{H}_{3}^{+}$astronomy. Rev Mod Phys 2020.

[234] Tóbiás R, Furtenbacher T, Császár AG, Naumenko OV, Tennyson J, Flaud JM, et al. Critical evaluation of measured rotational-vibrational transitions offour sulphur isotopologues of $\mathrm{S}^{16} \mathrm{O}_{2}$. J Quant Spectrosc Radiat Transf 2018;208:152-63. doi:10.1016/j.jqsit.2018.01.006.

[235] Chubb KL, Joseph M, Franklin J, Choudhury N, Furtenbacher T, Császár AG, et al. MARVEL Analysis of the measured high-resolution spectra of $\mathrm{C}_{2} \mathrm{H}_{2}$. J Quant Spectrosc Radiat Transf 2018;204:42-55. doi:10.1016/j.jqsrt.2017.08. 018.

[236] Al-Derzi AR, Furtenbacher T, Yurchenko SN, Tennyson J, Császár AG. MARVEL Analysis of the measured high-resolution spectra of formaldehyde. J Quant Spectrosc Radiat Transf 2020. To be submitted

[237] Al-Refaie AF, Ovsyannikov RI, Polyansky OL, Yurchenko SN, Tennyson J. A variationally calculated room temperature line-list for $\mathrm{H}_{2} \mathrm{O}_{2}$. J Mol Spectrosc 2015;318:84-90. doi:10.1016/j.jms.2015.10.004.

[238] Ito Y, Hashimoto GL, Takahashi YO, Ishiwatari M, Kuramoto $\mathrm{K} . \mathrm{H}_{2} \mathrm{O}_{2}$-induced greenhouse warming on oxidized early Mars. Astrophys J 2020;893:168. doi: $10.3847 / 1538-4357 / a b 7 d b 4$

[239] Bourgalais J, Carrasco N, Changeat Q Venot O, Javanović L, Pernot P, et al. Ions in the thermosphere of exoplanets: observational constraints revealed by innovative laboratory experiments. Astrophys J 2020;895:77. doi:10.3847/ 1538-4357/ab8e2d

[240] Sousa-Silva C, Seager S, Ranjan S, Petkowski JJ, Zhan Z, Hu R, et al. Phosphine as a biosignature gas in exoplanet atmospheres. Astrobiology 2020;20:23568. doi:10.1089/ast.2018.1954.

[241] Rey M, Nikitin AV, Tyuterev VG. Theoretical hot methane line list up $\mathrm{T}=2000$ for astrophysical applications. Astrophys J 2014;789:2. doi:10.1088/ 0004-637X/789/1/2.

[242] Hargreaves RJ, Bernath PF, Bailey J, Dulick M. Empirical line lists and absorption cross sections for methane at high temperatures. Astrophys J 2015;813:12.

[243] Rey M, Nikitin AV, Tyuterev VG. Accurate theoretical methane line lists in the infrared up to $3000 \mathrm{~K}$ and quasi-continuum absorption/emission modeling for astrophysical applications. Astrophys J 2017;847:105.

[244] Li G, Gordon IE, Hajigeorgiou PG, Coxon JA, Rothman LS. Reference spectroscopic data for hydrogen halides, part II: the line lists. J Quant Spectrosc Radiat Transf 2013;130:284-95. doi:10.1016/j.jqsrt.2013.07.019.

[245] Gamache RR. Total internal partition sums for 167 isotopologues of 53 molecules important in planetary atmospheres: application to HITRAN2016 and beyond. J Quant Spectrosc Radiat Transf 2017;203:70-87. doi:10.1016/j. jqsit.2017.03.045.

[246] Vallon R, Richard C, Crozet P, Wannous G, Ross A. Laboratory measurements of $\mathrm{NiH}$ by Fourier transform dispersed fluorescence. Astrophys J 2009;696:172-5. doi:10.1088/0004-637X/696/1/172.

[247] Harker H, Richard C, Tourasse G, Crozet P, Ross AJ. Zeeman spectroscopy of $\mathrm{NiH}$ : lande factors of three omega $=3 / 2$ excited electronic states. J Mol Spectrosc 2013;292:28-34. doi:10.1016/j.jms.2013.09.005

[248] Šimečková M, Jacquemart D, Rothman LS, Gamache RR, Goldman A. Einstein A coefficients and statistical weights for molecular absorption transitions in the HITRAN database. J Quant Spectrosc Radiat Transf 2006;98:130-55.
[249] Irwin AW. Polynomial partition function approximations of 344 atomic and molecular species. Astrophys J Suppl 1981;45:621-33. doi:10.1086/190730.

[250] Sauval AJ, Tatum JB. A set of partition functions and equilibrium constants for 300 diatomic molecules of astrophysical interest. Astrophys J Suppl 1984;56:193-209.

[251] Irwin AW. The partition functions of JANAF polyatomic molecules that significantly affect the stellar atmospheric equation of state. Astron Astrophys Suppl 1988;74:145-60.

[252] Barklem PS, Collet R. Partition functions and equilibrium constants for diatomic molecules and atoms of astrophysical interest. Astron Astrophys 2016;588:A96. doi:10.1051/0004-6361/201526961.

[253] Sousa-Silva C, Hesketh N, Yurchenko SN, Hill C, Tennyson J. High temperature partition functions and thermodynamic data for ammonia and phosphine. J Quant Spectrosc Radiat Transf 2014;142:66-74. doi:10.1016/j.jqsrt2014.03.012.

[254] Ranjan S., Schwieterman E.W., Harman C., Fateev A., Sousa-Silva C., Seager S., et al. Photochemistry of anoxic abiotic habitable planet atmospheres: Impact of new $\mathrm{H}_{2} \mathrm{O}$ cross-sections, Astrophys. J 896, 2020, 148.

[255] Fateev A, Clausen S, Wang Y, Yurchenko SN, Tennyson J. Experimental VUV cross sections in the ExoMol database. J Quant Spectrosc Radiat Transf 2020; In preparation.

[256] Venot O, Bénilan Y, Fray N, Gazeau MC, Lefévre F, Es-sebbar E, et al. VUVAbsorption cross section of carbon dioxide from 150 to $800 \mathrm{~K}$ and applications to warm exoplanetary atmospheres. Astron Astrophys 2018;609:A34. doi:10.1051/0004-6361/201731295.

[257] Tennyson J, Hill C, Yurchenko SN. Data structures for ExoMol: molecular line lists for exoplanet and other atmospheres. In: $6^{\text {th }}$ international conference on atomic and molecular data and their applications ICAMDATA-2012; vol. 1545 of AIP Conference Proceedings. AIP, New York; 2013. p. 186-95. Doi:10.1063/1.4815853

[258] Western CM. PGOPHER: A program for simulating rotational, vibrational and electronic spectra. J Quant Spectrosc Radiat Transf 2017;186:221-42. doi:10. 1016/j.jqsrt.2016.04.010.

[259] Furtenbacher T, Szidarovszky T, Hruby J, Kyuberis AA, Zobov NF, Polyansky OL, et al. Definitive high-temperature ideal-gas thermochemical functions of the $\mathrm{H}_{2}{ }^{16} \mathrm{O}$ molecule. J Phys Chem Ref Data 2016;45:043104. doi:10.1063/1. 4967723.

[260] Barton EJ, Hill C, Czurylo M, Li HY, Hyslop A, Yurchenko SN, et al. The ExoMol diet of line-by-line pressure-broadening parameters. J Quant Spectrosc Radiat Transf 2017;203:490-5. doi:10.1016/j.jqsit.2017.01.028.

[261] Fortney J.J., Robinson T.D., Domagal-Goldman S., Del Genio A.D., Gordon I.E., Gharib-Nezhad E., et al. The need for laboratory measurements and $\mathrm{ab}$ initio studies to aid understanding of exoplanetary atmospheres. 2019. ArXiv: 1905.07064

[262] Chubb KL, Rocchetto M, Yurchenko SN, Min M, Waldmann I, Barstow JK, et al. The ExoMolOP database: cross-sections and k-tables for molecules of interest in high-temperature exoplanet atmospheres. Astron Astrophys 2020.

[263] Dubernet ML, Boudon V, Culhane JL, Dimitrijevic MS, Fazliev AZ, Joblin C, et al. Virtual atomic and molecular data centre. J Quant Spectrosc Radiat Transf 2010:111:2151-9.

[264] Dubernet ML, Antony BK, Ba1 YA, Babikov YL, Bartschat K, Boudon V, et al. The virtual atomic and molecular data centre (VAMDC) consortium for astrophysics. J Phys B 2016:49:074003. doi:10.1088/0953-4075/49/7/074003.

[265] Gharib-Nezhad E, Iyer AR, Line MR, Freedman RS, Marley MS, Batalha NE. EXOPLINES: Molecular absorption cross-section database for brown dwarf and giant exoplanet atmospheres. Astrophys J Suppl 2020.

[266] Yurchenko SN, Al-Refaie AF, Tennyson J. ExoCross: a general program for generating spectra from molecular line lists. Astron Astrophys 2018;614:A131. doi:10.1051/0004-6361/201732531.

[267] Rothman LS, Gordon IE, Babikov Y, Barbe A, Benner DC, Bernath PF, et al The HITRAN 2012 molecular spectroscopic database. J Quant Spectrosc Radiat Transf 2013;130:4-50. doi:10.1016/jqsit.2013.07.002.

[268] Tennyson J, Bernath PF, Brown LR, Campargue A, Császár AG, Daumont L et al. IUPAC critical evaluation of the rotational-vibrational spectra of water vapor. part IV. energy levels and transition wavenumbers for $\mathrm{D}_{2}{ }^{16} \mathrm{O}, \mathrm{D}_{2}{ }^{17} \mathrm{O}$ and $\mathrm{D}_{2}{ }^{18}$ O. J Quant Spectrosc Radiat Transf 2014;142:93-108. doi:10.1016/j. jqsit.2014.03.019.

[269] Tóbiás R, Furtenbacher T, Tennyson J, Császár AG. Accurate empirical rovibrational energies and transitions of $\mathrm{H}_{2}{ }^{16} \mathrm{O}$. Phys Chem Chem Phys 2019;21:3473-95. doi:10.1039/c8cp05169k.

[270] Furtenbacher T, Tóbiás R, Tennyson J, Polyansky OL, Császár AG. W2020: A database of validated rovibrational experimental transitions and empirical energy levels of $\mathrm{H}_{2}{ }^{16}$ O. J Phys Chem Ref Data 2020;49:033101.

[271] Furtenbacher T, Szidarovszky T, Mátyus Edit Fábri C, Császár AG. Analysis of the rotational-vibrational states of the molecular ion $\mathrm{H}_{3}^{+}$. J Chem Theory Comput 2013;9:5471-8. doi:10.1021/ct4004355.

[272] Furtenbacher T, Coles PA, Tennyson J, Yurchenko SN, Yu S, Drouin B, et al. Empirical rovibational energy of ammonia up to $7500 \mathrm{~cm}^{-1}$. J Quant Spectrosc Radiat Transf 2020;251:107027. doi:10.1016/j.jqsrt.2020.107027.

[273] McKemmish LK, Borsovszky J, Goodhew KL, Sheppard S, Bennett AFV, Martin ADJ, et al. MARVEL Analysis of the measured high-resolution spectra of ${ }^{90} \mathrm{Zr}^{16}$ O. Astrophys J 2018;867:33. doi:10.3847/1538-4357/aadd19.

[274] Furtenbacher T, Horváth M, Koller D, Sólyom P, Balogh A, Balogh I, et al. MARVEL Analysis of the measured high-resolution rovibronic spectra and definitive ideal-gas thermochemistry of the ${ }^{16} \mathrm{O}_{2}$ molecule. J Phys Chem Ref Data 2019;48:023101. doi:10.1063/1.5083135. 
[275] Wang Y, Owens A, Tennyson J, Yurchenko SN. MARVEL Analysis of the measured high-resolution rovibronic spectra of the calcium monohydroxide radical (CaOH). Astrophys J Suppl 2020;248:9. doi:10.3847/1538-4365/ab85cb.

[276] Syme AM, McKemmish LK. Experimental energy levels of ${ }^{12} \mathrm{C}^{14} \mathrm{~N}$ through MARVEL analysis. Mon Not R Astron Soc 2020.

[277] de Kok RJ, Brogi M, Snellen IAG, Birkby J, Albrecht S, de Mooij EJW. Detection of carbon monoxide in the high-resolution day-side spectrum of the exoplanet HD 189733b. Astron Astrophys 2013;554:A82. doi:10.1051/0004-6361/ 201321381.

[278] Birkby JL, de Kok RJ, Brogi M, de Mooij EJW, Schwarz H, Albrecht S, et al. Detection of water absorption in the day side atmosphere of HD $189733 \mathrm{~b}$ using ground-based high-resolution spectroscopy at $3.2 \mu \mathrm{m}$. Mon Not R Astron Soc 2013;436:L35-9. doi:10.1093/mnrasl/slt107.

[279] Brogi M, de Kok RJ, Birkby JL, Schwarz H, Snellen IAG. Carbon monoxide and water vapor in the atmosphere of the non-transiting exoplanet HD $179949 \mathrm{~b}$. Astron Astrophys 2014;565:A124. doi:10.1051/0004-6361/201423537.

[280] Tennyson J. The ExoMol project: molecular opacity calculations at University College London. In: Mendoza C, Turck-Chiéze S, Colgan J, editors. Workshop on astrophysical opacities. Astron. Soc. Pac. Conf. Ser., vol. 515. Astronomical Society of the Pacific; 2018. p. 137-44.
[281] Furtenbacher T, Császár AG. MARVEL: Measured active rotational-vibrational energy levels. II. algorithmic improvements. J Quant Spectrosc Radiat Transf 2012:113:929-35.

[282] Darby-Lewis D, Shah H, Joshi D, Khan F, Kauwo M, Sethi N, et al. MARVEL analysis of the measured high-resolution spectra of NH. J Mol Spectrosc 2019;362:69-76. doi:10.1016/j.jms.2019.06.002.

[283] Sousa-Silva C, McKemmish LK, Chubb KL, Baker J, Barton EJ, Gorman MN, et al. Original research by young twinkle students (ORBYTS): when can students start performing original research? Phys Educ 2018;53:015020. doi:10 1088/1361-6552/aa8f2a.

[284] Skinner FM, Gordon IE, Hill C, Hargreaves RJ, Lockhart KE, Rothman LS. Referencing sources of molecular spectroscopic data in the era of data science: application to the HITRAN and AMBDAS databases. Atoms 2020;8:16. doi:10.3390/atoms8020016. 